\title{
Computing the ground state and dynamics of the nonlinear Schrödinger equation with nonlocal interactions via the nonuniform FFT
}

\author{
Weizhu Bao ${ }^{\mathrm{a}}$, Shidong Jiang ${ }^{\mathrm{b}}$, Qinglin Tang ${ }^{\mathrm{c}, \mathrm{d}}$, Yong Zhang ${ }^{\mathrm{e}, \mathrm{f}, *}$ \\ ${ }^{a}$ Department of Mathematics, National University of Singapore, Singapore 119076, Singapore \\ ${ }^{b}$ Department of Mathematical Sciences, New Jersey Institute of Technology, Newark, New Jersey, 07102, USA \\ ${ }^{c}$ Université de Lorraine, Institut Elie Cartan de Lorraine, UMR 7502, Vandoeuvre-lès-Nancy, F-54506, France \\ ${ }^{d}$ Inria Nancy Grand-Est/IECL-CORIDA, France \\ ${ }^{e}$ Wolfgang Pauli Institute c/o Fak. Mathematik, University Wien, Oskar-Morgenstern-Platz 1, 1090 Vienna, Austria \\ ${ }^{f}$ Beijing Computational Science Research Center, Beijing 100084, P. R. China
}

\begin{abstract}
We present efficient and accurate numerical methods for computing the ground state and dynamics of the nonlinear Schrödinger equation (NLSE) with nonlocal interactions based on a fast and accurate evaluation of the long-range interactions via the nonuniform fast Fourier transform (NUFFT). We begin with a review of the fast and accurate NUFFT based method in [29] for nonlocal interactions where the singularity of the Fourier symbol of the interaction kernel at the origin can be canceled by switching to spherical or polar coordinates. We then extend the method to compute other nonlocal interactions whose Fourier symbols have stronger singularity at the origin that cannot be canceled by the coordinate transform. Many of these interactions do not decay at infinity in the physical space, which adds another layer of complexity since it is more difficult to impose the correct artificial boundary conditions for the truncated bounded computational domain. The performance of our method against other existing methods is illustrated numerically, with particular attention on the effect of the size of the computational domain in the physical space. Finally, to study the ground state and dynamics of the NLSE, we propose efficient and accurate numerical methods by combining the NUFFT method for potential evaluation with the normalized gradient flow using backward Euler Fourier pseudospectral discretization and time-splitting Fourier pseudospectral method, respectively. Extensive numerical comparisons are carried out between these methods and other existing methods for computing the ground state and dynamics of the NLSE with various nonlocal interactions. Numerical results show that our scheme performs much better than those existing methods in terms of both accuracy and efficiency.
\end{abstract}

Keywords: nonlinear Schrödinger equation, nonlocal interactions, nonuniform FFT, ground state, dynamics, Poisson equation, fractional Poisson equation

\section{Introduction}

In this paper, we present efficient and accurate numerical methods and compare them with existing numerical methods for computing the ground state and dynamics of the nonlinear Schrödinger equation (NLSE). In dimensionless form, the NLSE with a nonlocal (long-range) interaction in $d$-dimensions $(d=$

\footnotetext{
* Corresponding author.

Email addresses: matbaowz@nus.edu.sg (Weizhu Bao), shidong.jiang@njit.edu (Shidong Jiang), qinglin.tang@inria.fr (Qinglin Tang), yong.zhang@univie.ac.at (Yong Zhang)

URL: http://www.math.nus.edu.sg/ bao/ (Weizhu Bao)
} 
$3,2,1)$ is

$$
\begin{aligned}
i \partial_{t} \psi(\mathbf{x}, t) & =\left[-\frac{1}{2} \Delta+V(\mathbf{x})+\beta \varphi(\mathbf{x}, t)\right] \psi(\mathbf{x}, t), \quad \mathbf{x} \in \mathbb{R}^{d}, \quad t>0, \\
\varphi(\mathbf{x}, t) & =\left(U *|\psi|^{2}\right)(\mathbf{x}, t), \quad \quad \mathbf{x} \in \mathbb{R}^{d}, \quad t \geq 0 ;
\end{aligned}
$$

with the initial data

$$
\psi(\mathbf{x}, t=0)=\psi_{0}(\mathbf{x}), \quad \mathbf{x} \in \mathbb{R}^{d} .
$$

Here, $t$ is time, $\mathbf{x}$ is the spatial coordinates, $\psi(\mathbf{x}, t)$ is the complex-valued wave-function, $V(\mathbf{x})$ is a given real-valued external potential, $\beta$ is a dimensionless interaction constant (positive for repulsive interaction and negative for attractive interaction), and $\varphi(\mathbf{x}, t)$ is a real-valued nonlocal (long-range) interaction which is defined as the convolution of an interaction kernel $U(\mathbf{x})$ and the density function $\rho(\mathbf{x}, t)=|\psi(\mathbf{x}, t)|^{2}$. The NLSE with the nonlocal interaction (1.1)-(1.2) has been widely used in modelling a variety of problems arising from quantum physics and chemistry to materials science and biology. It is nonlinear, dispersive and time transverse invariant, i.e., if $V(\mathbf{x}) \rightarrow V(\mathbf{x})+\alpha$ and $\varphi(\mathbf{x}, t) \rightarrow \varphi(\mathbf{x}, t)+\delta$, then $\psi(\mathbf{x}, t) \rightarrow \psi(\mathbf{x}, t) e^{-i(\alpha+\beta \delta) t}$, which immediately implies that the physical observables such as the density $\rho(\mathbf{x}, t)$ are kept unchanged. In addition, it conserves the mass and energy defined as follows:

$$
\begin{aligned}
& N(\psi(\cdot, t)):=\int_{\mathbb{R}^{d}}|\psi(\mathbf{x}, t)|^{2} d \mathbf{x} \equiv \int_{\mathbb{R}^{d}}|\psi(\mathbf{x}, 0)|^{2} d \mathbf{x}=\int_{\mathbb{R}^{d}}\left|\psi_{0}(\mathbf{x})\right|^{2} d \mathbf{x}=N\left(\psi_{0}\right), \quad t \geq 0, \\
& E(\psi(\cdot, t)):=\int_{\mathbb{R}^{d}}\left[\frac{1}{2}|\nabla \psi(\mathbf{x}, t)|^{2}+V(\mathbf{x})|\psi(\mathbf{x}, t)|^{2}+\frac{1}{2} \beta \varphi(\mathbf{x}, t)|\psi(\mathbf{x}, t)|^{2}\right] d \mathbf{x} \equiv E\left(\psi_{0}\right) .
\end{aligned}
$$

One of the most important nonlocal interactions in applications is the Coulomb interaction whose interaction kernel in $3 \mathrm{D} / 2 \mathrm{D}$ is given as

$$
U_{\mathrm{Cou}}(\mathbf{x})=\left\{\begin{array}{l}
\frac{1}{4 \pi|\mathbf{x}|}, \\
\frac{1}{2 \pi|\mathbf{x}|},
\end{array} \quad \widehat{U}_{\mathrm{Cou}}(\mathbf{k})=\left\{\begin{array}{ll}
\frac{1}{|\mathbf{k}|^{2}}, & d=3, \\
\frac{1}{|\mathbf{k}|}, & d=2,
\end{array} \quad \mathbf{x}, \mathbf{k} \in \mathbb{R}^{d},\right.\right.
$$

where $\widehat{f}(\mathbf{k})=\int_{\mathbb{R}^{d}} f(\mathbf{x}) e^{-i \mathbf{k} \cdot \mathbf{x}} d \mathbf{x}$ is the Fourier transform of $f(\mathbf{x})$ for $\mathbf{x}, \mathbf{k} \in \mathbb{R}^{d}$. In 3D, the Coulomb interaction kernel $U_{\mathrm{Cou}}(\mathbf{x})$ is exactly the Green's function of the Laplace operator and thus the nonlocal Coulomb interaction $\varphi$ in (1.2) also satisfies the Poisson equation in 3D

$$
-\Delta \varphi(\mathbf{x}, t)=|\psi(\mathbf{x}, t)|^{2}, \quad \mathbf{x} \in \mathbb{R}^{3}, \quad \lim _{|\mathbf{x}| \rightarrow \infty} \varphi(\mathbf{x}, t)=0, \quad t \geq 0 .
$$

In this case, (1.1)-(1.2) is also referred as the 3D Schrödinger-Poisson system (SPS) which was derived from the linear Schrödinger equation for a many-body (e.g., $N$ electrons) quantum system with binary Coulomb interaction between different electrons via the "mean field limit" [12, 13, 23]. It has important applications in modelling semiconductor devices and calculating electronic structures in materials simulation and design. On the other hand, the Coulomb interaction kernel $U(\mathbf{x})$ in 2D is the Green's function of the square-rootLaplace operator instead of the Laplace operator and thus the nonlocal Coulomb interaction $\varphi$ in (1.2) also satisfies the fractional Poisson equation in $2 \mathrm{D}$

$$
\sqrt{-\Delta} \varphi(\mathbf{x}, t)=|\psi(\mathbf{x}, t)|^{2}, \quad \mathbf{x} \in \mathbb{R}^{2}, \quad \lim _{|\mathbf{x}| \rightarrow \infty} \varphi(\mathbf{x}, t)=0, \quad t \geq 0 .
$$

In this case, (1.1)-(1.2) could be obtained from the 3D SPS under an infinitely strong external confinement in the $z$-direction $[9,14]$. This model could be used for modelling 2D materials such as graphene and "electron sheets" [20].

Another type of interaction from applications is that the interaction kernel $U(\mathbf{x})$ is taken as the Green's function of the Laplace operator in $3 \mathrm{D} / 2 \mathrm{D} / 1 \mathrm{D}[41]$

$$
U_{\text {Lap }}(\mathbf{x})=\left\{\begin{array}{ll}
\frac{1}{4 \pi|\mathbf{x}|}, & d=3, \\
-\frac{1}{2 \pi} \ln |\mathbf{x}|, & d=2, \\
-\frac{1}{2}|\mathbf{x}|, & d=1,
\end{array} \quad \Longleftrightarrow \quad \widehat{U}_{\text {Lap }}(\mathbf{k})=\frac{1}{|\mathbf{k}|^{2}}, \quad \mathbf{x}, \mathbf{k} \in \mathbb{R}^{d} .\right.
$$


When $d=3, U_{\text {Lap }}(\mathbf{x})=U_{\text {Cou }}(\mathbf{x})$ for $\mathbf{x} \in \mathbb{R}^{3}$. When $d=2$, the nonlocal interaction $\varphi$ in (1.2) with (1.9) satisfies the Poisson equation in $2 \mathrm{D}$ with the far-field condition

$$
-\Delta \varphi(\mathbf{x}, t)=|\psi(\mathbf{x}, t)|^{2}, \quad \mathbf{x} \in \mathbb{R}^{2}, \quad \lim _{|\mathbf{x}| \rightarrow \infty}\left[\varphi(\mathbf{x}, t)+\frac{C_{0}}{2 \pi} \ln |\mathbf{x}|\right]=0, \quad t \geq 0
$$

and when $d=1$ with $\mathbf{x}=x$, it satisfies the Poisson equation in $1 \mathrm{D}$ with the far-field condition

$$
-\partial_{x x} \varphi(x, t)=|\psi(x, t)|^{2}, \quad x \in \mathbb{R}, \quad \lim _{x \rightarrow \pm \infty}\left[\varphi(x, t)+\frac{1}{2}\left(C_{0}|x| \mp C_{1}\right)\right]=0, \quad t \geq 0,
$$

where $C_{0}=\int_{\mathbb{R}^{d}}|\psi(\mathbf{x}, t)|^{2} d \mathbf{x}=\widehat{|\psi|^{2}}(\mathbf{0}, t) \equiv \int_{\mathbb{R}^{d}}\left|\psi_{0}(\mathbf{x})\right|^{2} d \mathbf{x}=\widehat{\left|\psi_{0}\right|^{2}}(\mathbf{0})=N\left(\psi_{0}\right)$ and $C_{1}=\int_{\mathbb{R}} x|\psi(x, t)|^{2} d x=$ $\left.\widehat{\left(x|\psi|^{2}\right.}\right)(\mathbf{0}, t)$, which indicate that the nonlocal interaction $\varphi(\mathbf{x}, t) \rightarrow-\infty$ as $|\mathbf{x}| \rightarrow \infty$ in 2D/1D. In fact, when $d=2$ or $d=1,(1.1)-(1.2)$ with (1.9) is also referred as the 2D or 1D SPS. They could be obtained from the 3D SPS by integrating the 3D Coulomb interaction kernel $U_{\mathrm{Cou}}(\mathbf{x})$ along the $z$-line or $(y, z)$-plane under the assumption that the electrons are uniformly distributed in one or two spatial dimensions, respectively. The 2D/1D SPS is usually used for modelling 2D "electron sheets" and 1D "quantum wires", respectively, as well as lower dimensions semiconductor devices [32].

Recently, the following nonlocal interaction kernels in 2D/1D were obtained from the 3D SPS under strongly confining external potentials in the $z$-direction and $(y, z)$-plane, respectively

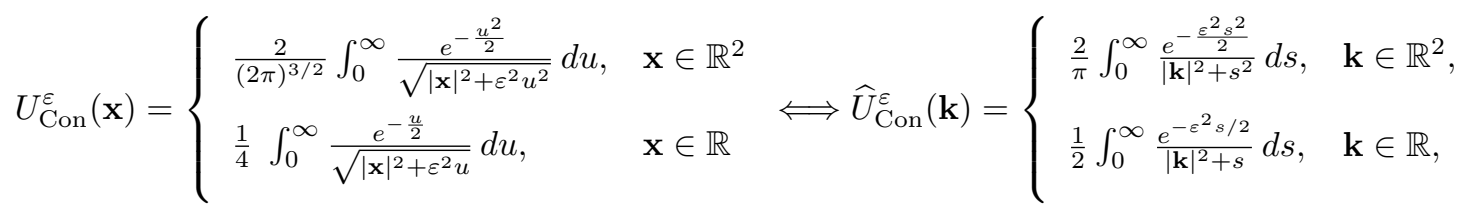

where $0<\varepsilon \ll 1$ is a dimensionless constant describing the ratio of the anisotropic confinement in different directions in the original 3D SPS [9]. In this case, the convolution (1.2) for the nonlocal interaction $\varphi$ can no longer be re-formulated into a partial differential equation. For other nonlocal interactions considered in quantum chemistry and dipole Bose-Einstein condensation, e.g., the dipole-dipole interaction, we refer to $[4,5,17,29]$ and references therein.

The ground state $\phi_{g}$ of the NLSE is defined as follows:

$$
\phi_{g}=\underset{\phi \in S}{\operatorname{argmin}} E(\phi), \quad \text { where } \quad S:=\left\{\left.\phi(\mathbf{x})\left|\|\phi\|^{2}:=\int_{\mathbb{R}^{d}}\right| \phi(\mathbf{x})\right|^{2} d \mathbf{x}=1, E(\phi)<\infty\right\} .
$$

For the existence, uniqueness and properties of the ground state as well as the well-posedness and dynamical properties of the NLSE, we refer to $[36,18,15,4,14,19,33,34]$ and references therein.

In order to numerically compute the ground state of (1.13) and the dynamics of (1.1)-(1.2), one of the key difficulties is to efficiently and accurately evaluate the nonlocal interaction (1.2) for a given density $\rho=|\psi|^{2}$. Note that

$$
\varphi(\mathbf{x}, t)=\int_{\mathbb{R}^{d}} U(\mathbf{x}-\mathbf{y}) \rho(\mathbf{y}, t) d \mathbf{y}=\frac{1}{(2 \pi)^{d}} \int_{\mathbb{R}^{d}} \widehat{U}(\mathbf{k}) \widehat{\rho}(\mathbf{k}, t) e^{i \mathbf{k} \cdot \mathbf{x}} d \mathbf{k}, \quad \mathbf{x} \in \mathbb{R}^{d}, \quad t \geq 0,
$$

it is natural to evaluate $\varphi(\mathbf{x}, t)$ via the standard fast Fourier transform (FFT) using a uniform grid on a bounded computational domain $\Omega$ [11]. However, the accuracy of this approach is hampered due to the singularity of $\widehat{U}(\mathbf{k})$ at the origin. Indeed, for the 3D Coulomb interaction, this method is equivalent to solving the Poisson equation (1.7) on $\Omega$ with periodic boundary condition via the Fourier spectral method. It is easy to see that this approach is inconsistent due to the inappropriate periodic boundary condition:

$$
0<\int_{\Omega}|\psi(\mathbf{x}, t)|^{2} d \mathbf{x}=-\int_{\Omega} \Delta \varphi(\mathbf{x}, t) d \mathbf{x}=-\int_{\partial \Omega} \frac{\partial \varphi}{\partial \mathrm{n}} d s=0
$$


Thus, this approach suffers from no convergence in terms of the mesh size of partitioning any fixed $\Omega$ (a phenomenon known as "numerical locking" in the literature); and its convergence is very slow, e.g., linearly convergent for the $3 \mathrm{D} / 2 \mathrm{D}$ Coulomb interaction, in terms of the size of $\Omega$ because $\varphi$ decays like $\frac{1}{|\mathbf{x}|}$. To overcome this "numerical locking", a numerical method was proposed by imposing the homogeneous Dirichlet boundary condition on $\partial \Omega$, and then solving the truncated problem via the discrete sine transform (DST) $[6,17,41]$. This method avoids numerically the singularity of $\widehat{U}(\mathbf{k})$ at the origin and thus significantly improves the accuracy in the evaluation of the Coulomb interaction potential. However, the truncation error of this method still decays only linearly in terms of the size of $\Omega$ due to the slow decaying property of the Coulomb potential. Thus when high accuracy is required, the bounded computational domain $\Omega$ must be chosen very large, which increases significantly the computational cost in both memory and CPU time for evaluating the nonlocal interaction potential (1.2) and solving the NLSE (1.1). Moreover, for the purpose of solving the NLSE, a much smaller computational domain actually suffices since the wave-function $\psi$ decays exponentially fast when $|\mathbf{x}| \rightarrow \infty$ in most applications. We would also like to point out that this method could not be extended to the cases where the potential in (1.1) either does not decay at infinity (for example, $1 \mathrm{D} / 2 \mathrm{D}$ cases of $(1.9)$ ) or cannot be converted to a PDE problem (as in (1.12)).

Recently, a fast and accurate NUFFT based method [29] was proposed to compute the Coulomb interaction (1.6) in 3D/2D. The key observation there is that the singularity in the Fourier transform of the interaction kernel $\widehat{U}(\mathbf{k})$ at the origin can be canceled out with the Jacobian in spherical or polar coordinates, thus making the integrand in (1.14) smooth. The integral is then approximated via a high-order quadrature and the resulting discrete summation is evaluated via the NUFFT. The algorithm has $O(N \log N)$ complexity with $N$ the total number of unknowns in the physical space and achieves very high accuracy for the evaluation of Coulomb interactions [29]. The main aims of this paper are fourfold: (i) to extend the algorithm in [29] to evaluate the nonlocal interactions whose Fourier symbols have stronger singularity at the origin which cannot be canceled by coordinate transform; (ii) to compare numerically the newly developed NUFFT based method with those existing methods for nonlocal interaction evaluation; (iii) to propose efficient and accurate numerical methods for computing the ground state and dynamics of the NLSE (1.1)-(1.2) by incorporating the NUFFT based algorithm into the normalized gradient flow method and the time-splitting Fourier pseudospectral method, respectively, and (iv) to compare these two new schemes with those existing ones for computing the ground state and dynamics of the NLSE.

The paper is organized as follows. In Section 2, the NUFFT based algorithm in [29] for the evaluation of Coulomb interaction is reviewed and then extended for the case of general nonlocal interaction (1.2) whose kernel is taken as either (1.9) or (1.12). Then, efficient and accurate numerical methods are proposed respectively in Section 3 and Section 4 to compute the ground state and dynamics of the NLSE (1.1)-(1.2). Finally, some concluding remarks are drawn in Section 5.

\section{An algorithm for the evaluation of the nonlocal interaction via the NUFFT}

In this section, we will propose a fast and accurate evaluation of the nonlocal interaction

$$
u(\mathbf{x})=(U * \rho)(\mathbf{x})=\frac{1}{(2 \pi)^{d}} \int_{\mathbb{R}^{d}} \widehat{U}(\mathbf{k}) \widehat{\rho}(\mathbf{k}) e^{i \mathbf{k} \cdot \mathbf{x}} d \mathbf{k}, \quad \mathbf{x} \in \mathbb{R}^{d}, \quad d=3,2,1,
$$

where $\rho(\mathbf{x})$ satisfying $\widehat{\rho}(\mathbf{0})=\int_{\mathbb{R}^{d}} \rho(\mathbf{x}) d \mathbf{x}>0$ is a given nonnegative smooth density function that decays rapidly at far field. We will first briefly review the algorithm in [29] for fast and accurate evaluation of the Coulomb interactions, and then extend it to the cases where $U(\mathbf{x})$ in (2.1) is taken as either (1.9) or (1.12).

\subsection{Coulomb interactions in $3 D / 2 D$}

When $U(\mathbf{x})$ reads as (1.6), by truncating the integration domain in (2.1) into a bounded domain and adopting the spherical/polar coordinates in 3D/2D in the Fourier (or phase) space, we have [29] 


$$
\begin{aligned}
u(\mathbf{x}) & =\frac{1}{(2 \pi)^{d}} \int_{\mathbb{R}^{d}} e^{i \mathbf{k} \cdot \mathbf{x}} \widehat{U}_{\mathrm{Cou}}(\mathbf{k}) \widehat{\rho}(\mathbf{k}) d \mathbf{k}=\frac{1}{(2 \pi)^{d}} \int_{\mathbb{R}^{d}} \frac{1}{|\mathbf{k}|^{d-1}} e^{i \mathbf{k} \cdot \mathbf{x}} \widehat{\rho}(\mathbf{k}) d \mathbf{k} \\
& \approx \frac{1}{(2 \pi)^{d}} \int_{|\mathbf{k}| \leq P} \frac{1}{|\mathbf{k}|^{d-1}} e^{i \mathbf{k} \cdot \mathbf{x}} \widehat{\rho}(\mathbf{k}) d \mathbf{k} \\
& =\frac{1}{(2 \pi)^{d}} \begin{cases}\int_{0}^{P} \int_{0}^{\pi} \int_{0}^{2 \pi} e^{i \mathbf{k} \cdot \mathbf{x}} \widehat{\rho}(\mathbf{k}) \sin \theta d|\mathbf{k}| d \theta d \phi, & d=3, \\
\int_{0}^{P} \int_{0}^{2 \pi} e^{i \mathbf{k} \cdot \mathbf{x}} \widehat{\rho}(\mathbf{k}) d|\mathbf{k}| d \phi, & \mathbf{x} \in \Omega \subset \mathbb{R}^{d}\end{cases}
\end{aligned}
$$

Here, $P=O\left(1 / \varepsilon_{0}\right)^{1 / n}, \varepsilon_{0}>0$ is the prescribed precision (e.g., $\left.\varepsilon_{0}=10^{-10}\right)$, and $n$ is the decaying rate of $\widehat{\rho}(\mathbf{k})$ at infinity (i.e., $\widehat{\rho}(\mathbf{k})=O\left(|\mathbf{k}|^{-n}\right)$ as $|\mathbf{k}| \rightarrow \infty$ ). Correspondingly, we choose a bounded domain $\Omega$ large enough such that the truncation error of $\rho(\mathbf{x})$ is negligible. It is easy to see that the singularity of the integrand at the origin in phase space is removed in spherical or polar coordinates. Thus, the above integral can be discretized using high order quadratures and the resulting summation can be evaluated efficiently via the NUFFT. This leads to an $O(N \log N)+O(M)$ algorithm where $N$ is the total number of equispaced points in the physical space and $M$ is the number of nonequispaced points in the Fourier space. However, although $M$ is roughly the same order as $N$, the constant in front of $O(M)$ (e.g., $24^{d}$ for 12-digit accuracy) is much greater than the constant in front of $O(N \log N)$. This makes the algorithm considerably slower than the regular FFT, especially for three dimensional problems.

An improved algorithm is developed to reduce the computational cost in [29]. First, the integral in (2.2) is further split into two parts via a simple partition of unity:

$$
\begin{aligned}
u(\mathbf{x}) & \approx \frac{1}{(2 \pi)^{d}} \int_{|\mathbf{k}| \leq P} \frac{1}{|\mathbf{k}|^{d-1}} e^{i \mathbf{k} \cdot \mathbf{x}} \widehat{\rho}(\mathbf{k}) d \mathbf{k} \\
& =\frac{1}{(2 \pi)^{d}} \int_{|\mathbf{k}| \leq P} e^{i \mathbf{k} \cdot \mathbf{x}} \frac{1-p_{d}(\mathbf{k})}{|\mathbf{k}|^{d-1}} \widehat{\rho}(\mathbf{k}) d \mathbf{k}+\frac{1}{(2 \pi)^{d}} \int_{|\mathbf{k}| \leq P} e^{i \mathbf{k} \cdot \mathbf{x}} \frac{p_{d}(\mathbf{k})}{|\mathbf{k}|^{d-1}} \widehat{\rho}(\mathbf{k}) d \mathbf{k} \\
& \approx \frac{1}{(2 \pi)^{d}} \int_{\mathcal{D}} e^{i \mathbf{k} \cdot \mathbf{x}} w_{d}(\mathbf{k}) \widehat{\rho}(\mathbf{k}) d \mathbf{k}+\frac{1}{(2 \pi)^{d}} \int_{|\mathbf{k}| \leq P} e^{i \mathbf{k} \cdot \mathbf{x}} \frac{p_{d}(\mathbf{k})}{|\mathbf{k}|^{d-1}} \widehat{\rho}(\mathbf{k}) d \mathbf{k}:=I_{1}+I_{2}, \quad \mathbf{x} \in \Omega
\end{aligned}
$$

Here, $\mathcal{D}=\left\{\mathbf{k}=\left(k_{1}, \ldots, k_{d}\right)^{T} \mid-P \leq k_{j} \leq P, j=1, \ldots, d\right\}$ is a rectangular domain containing the ball $B$, the function $p_{d}(\mathbf{k})$ is chosen such that it is a $C^{\infty}$ function that decays exponentially fast as $|\mathbf{k}| \rightarrow \infty$ and the function $w_{d}(\mathbf{k}):=\frac{1-p_{d}(\mathbf{k})}{|\mathbf{k}|^{d-1}}$ is smooth for $\mathbf{k} \in \mathbb{R}^{d}$. We remark here that similar ideas by partition of unity to remove the singularity were also used for surface integral [16].

With this $p_{d}(\mathbf{k}), I_{1}$ can be computed via the regular FFT and $I_{2}$ can be evaluated via the NUFFT with a fixed (much fewer) number of irregular points in the Fourier space (see Figure 1). Thus the interpolation cost in the NUFFT is reduced to $O(1)$ and the cost of the overall algorithm is comparable to that of the regular FFT, with an oversampling factor $\left(2^{3}\right.$ for $3 \mathrm{D}$ problems and $2^{2}-3^{2}$ for $2 \mathrm{D}$ problems $)$ in front of $O(N \log N)$.

\subsection{Poisson potentials in $2 D / 1 D$}

When $U(\mathbf{x})$ is taken as $(1.9)$ in $2 \mathrm{D} / 1 \mathrm{D}$, the algorithm discussed in the previous section cannot be applied directly to evaluate the Poisson potential $u(\mathbf{x})$ due to the stronger singularity of $\widehat{U}_{\text {Lap }}(\mathbf{k})$ at the origin. Obviously, the Poisson potential $u(\mathbf{x})$ satisfies the Poisson equation $-\Delta u(\mathbf{x})=\rho(\mathbf{x})$ with the far field condition

$$
\lim _{|\mathbf{x}| \rightarrow \infty}\left[u(\mathbf{x})+\frac{\widehat{\rho}(\mathbf{0})}{2 \pi} \ln |\mathbf{x}|\right]=0
$$

for $2 \mathrm{D}$ problems and

$$
\lim _{x \rightarrow \pm \infty}\left[u(x)+\frac{1}{2}(\widehat{\rho}(0)|x| \mp \widehat{(x \rho)}(0))\right]=0
$$




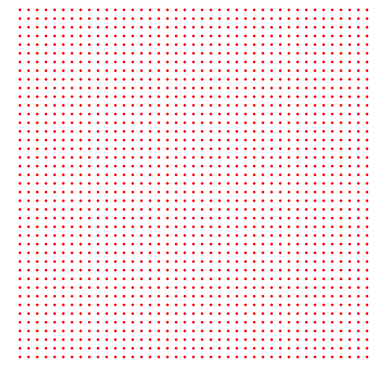

(a) Regular grid

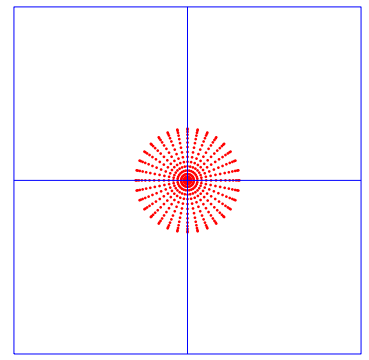

(b) Polar grid

Figure 1: Two grids used in the Fourier domain in the improved algorithm in [29]: the regular grid on the left panel is used to compute $I_{1}$ in (2.3) via the regular FFT; while the polar grid (confined in a small region centered at the origin) on the right panel is used to compute $I_{2}$ in (2.3) via the NUFFT. Note that the number of points in the polar grid is $O(1)$, thus keeping the interpolation cost in NUFFT minimal.

for $1 \mathrm{D}$ problems, respectively.

Let us first consider the evaluation of the 2D Poisson potential. To overcome the above mentioned difficulties, we introduce the auxiliary functions

$$
G(\mathbf{x})=\frac{1}{2 \pi \sigma^{2}} e^{-\frac{|\mathbf{x}|^{2}}{2 \sigma^{2}}}, \quad G_{1}(\mathbf{x})=\widehat{\rho}(\mathbf{0}) G(\mathbf{x})-\widehat{(\mathbf{x} \rho)}(\mathbf{0}) \cdot \nabla_{\mathbf{x}} G(\mathbf{x}), \quad \mathbf{x} \in \mathbb{R}^{2},
$$

and the function $u_{1}(\mathbf{x})$ which satisfies the Poisson equation with the far-field condition:

$$
-\Delta u_{1}(\mathbf{x})=G_{1}(\mathbf{x}), \quad \mathbf{x} \in \mathbb{R}^{2}, \quad \lim _{|\mathbf{x}| \rightarrow \infty}\left[u_{1}(\mathbf{x})+\frac{\widehat{\rho}(\mathbf{0})}{2 \pi} \ln |\mathbf{x}|\right]=0 .
$$

Here, $\sigma>0$ is a parameter to be chosen later. Solving (2.7) via the convolution, we have

$$
u_{1}(\mathbf{x})=\left(U_{\text {Lap }} * G_{1}\right)(\mathbf{x})=\widehat{\rho}(\mathbf{0}) u_{1,1}(\mathbf{x})-\widehat{(\mathbf{x} \rho)}(\mathbf{0}) \cdot \mathbf{u}_{1,2}(\mathbf{x}), \quad \mathbf{x} \in \mathbb{R}^{2},
$$

where

$$
u_{1,1}(\mathbf{x})=\left(U_{\text {Lap }} * G\right)(\mathbf{x}), \quad \quad \mathbf{u}_{1,2}(\mathbf{x})=\nabla_{\mathbf{x}} u_{1,1}(\mathbf{x}), \quad \mathbf{x} \in \mathbb{R}^{2}
$$

Note that $G(\mathbf{x})$ is radially symmetric, i.e., $G(\mathbf{x})=G(|\mathbf{x}|)=G(r)$ with $r=|\mathbf{x}| \geq 0$ and $u_{1,1}(\mathbf{x})$ satisfies the Poisson equation

$$
-\Delta u_{1,1}(\mathbf{x})=G(\mathbf{x}), \quad \mathbf{x} \in \mathbb{R}^{2}, \quad \lim _{|\mathbf{x}| \rightarrow \infty}\left[u_{1,1}(\mathbf{x})+\frac{1}{2 \pi} \ln |\mathbf{x}|\right]=0 .
$$

It is clear that $u_{1,1}(\mathbf{x})$ is also radially symmetric, i.e., $u_{1,1}(\mathbf{x})=u_{1,1}(r)$. Thus, the Poisson equation (2.10) can be re-formulated as the following second order ODE:

$$
-\frac{1}{r} \partial_{r}\left(r \partial_{r} u_{1,1}(r)\right)=G(r), \quad 0<r<\infty, \quad \lim _{r \rightarrow \infty}\left[u_{1,1}(r)+\frac{1}{2 \pi} \ln r\right]=0 .
$$

Integrating the above ODE twice with the far-field boundary condition, we obtain

$$
u_{1,1}(\mathbf{x})=\left\{\begin{array}{ll}
-\frac{1}{4 \pi}\left[\mathrm{E}_{1}\left(\frac{|\mathbf{x}|^{2}}{2 \sigma^{2}}\right)+2 \ln (|\mathbf{x}|)\right], & \mathbf{x} \neq \mathbf{0}, \\
\frac{1}{4 \pi}\left(\gamma_{e}-\ln \left(2 \sigma^{2}\right)\right), & \mathbf{x}=\mathbf{0},
\end{array} \quad \mathbf{x} \in \mathbb{R}^{2},\right.
$$


where $\mathrm{E}_{1}(r):=\int_{r}^{\infty} t^{-1} e^{-t} \mathrm{~d} t$ for $r>0$ is the exponential integral function [1] and $\gamma_{e} \approx 0.5772156649015328606$ is the Euler-Mascheroni constant. Differentiating (2.12) leads to

$$
\mathbf{u}_{1,2}(\mathbf{x})=\left\{\begin{array}{ll}
-\frac{1}{2 \pi} \frac{\mathbf{x}}{|\mathbf{x}|^{2}}\left(1-e^{-\frac{|\mathbf{x}|^{2}}{2 \sigma^{2}}}\right), & \mathbf{x} \neq \mathbf{0}, \\
0, & \mathbf{x}=\mathbf{0},
\end{array} \quad \mathbf{x} \in \mathbb{R}^{2}\right.
$$

Denote

We have

$$
u_{2}(\mathbf{x})=u(\mathbf{x})-u_{1}(\mathbf{x}) \quad \Longleftrightarrow \quad u(\mathbf{x})=u_{1}(\mathbf{x})+u_{2}(\mathbf{x}), \quad \mathbf{x} \in \mathbb{R}^{2}
$$

$$
-\Delta u_{2}(\mathbf{x})=\rho(\mathbf{x})-G_{1}(\mathbf{x}), \quad \mathbf{x} \in \mathbb{R}^{2}, \quad \lim _{|\mathbf{x}| \rightarrow \infty} u_{2}(\mathbf{x})=0 .
$$

Solving the above problem via the Fourier integral, noticing (2.6) and using the fact that

$$
\nabla_{\mathbf{k}} \widehat{\rho}(\mathbf{0})=-i \widehat{(\mathbf{x} \rho)}(\mathbf{0})=-i \int_{\mathbb{R}^{2}} \mathbf{x} \rho(\mathbf{x}) d \mathbf{x}
$$

we obtain

$$
\begin{aligned}
u_{2}(\mathbf{x}) & =\left(U_{\text {Lap }} *\left(\rho-G_{1}\right)\right)(\mathbf{x})=\frac{1}{(2 \pi)^{2}} \int_{\mathbb{R}^{2}} \frac{\widehat{\rho}(\mathbf{k})-\widehat{G_{1}}(\mathbf{k})}{|\mathbf{k}|^{2}} e^{i \mathbf{k} \cdot \mathbf{x}} d \mathbf{k} \\
& =\frac{1}{(2 \pi)^{2}} \int_{\mathbb{R}^{2}} \frac{W(\mathbf{k})}{|\mathbf{k}|} e^{i \mathbf{k} \cdot \mathbf{x}} d \mathbf{k} \approx \frac{1}{(2 \pi)^{2}} \int_{0}^{P} \int_{0}^{2 \pi} W(\mathbf{k}) e^{i \mathbf{k} \cdot \mathbf{x}} d|\mathbf{k}| d \theta, \quad \mathbf{x} \in \Omega \subset \mathbb{R}^{2},
\end{aligned}
$$

where

$$
W(\mathbf{k})=\left\{\begin{array}{lll}
\frac{\widehat{\rho}(\mathbf{k})-\widehat{G_{1}}(\mathbf{k})}{|\mathbf{k}|}=\frac{\widehat{\rho}(\mathbf{k})-\left(\widehat{\rho}(\mathbf{0})+\mathbf{k} \cdot \nabla_{\mathbf{k}} \widehat{\rho}(\mathbf{0})\right) e^{-\frac{1}{2}|\mathbf{k}|^{2} \sigma^{2}}}{|\mathbf{k}|}, & \mathbf{k} \neq \mathbf{0}, & \mathbf{k} \in \mathbb{R}^{2} . \\
0, & \mathbf{k}=\mathbf{0}, &
\end{array}\right.
$$

Note that the singularity of $W(\mathbf{k}) /|\mathbf{k}|$ at the origin in (2.16) is removed by switching to polar coordinates in the Fourier space, and thus $u_{2}(\mathbf{x})$ can be evaluated by the algorithm in [29].

In practical computations, the parameter $\sigma$ in (2.6) should be chosen appropriately such that the Gaussian $e^{-\frac{1}{2}|\mathbf{k}|^{2} \sigma^{2}}$ and $\mathbf{k} \cdot \nabla_{\mathbf{k}} \widehat{\rho}(\mathbf{0}) e^{-\frac{1}{2}|\mathbf{k}|^{2} \sigma^{2}}$ in the Fourier space decay at the same rate or faster than $\widehat{\rho}(\mathbf{k})$ when $|\mathbf{k}|$ is large. With this choice of $\sigma$, there is no need to enlarge the computational domain in the Fourier space for the evaluation of (2.16) via the NUFFT. On the other hand, there is no need to oversample the truncated Fourier domain due to the rapid decaying of the Gaussian $e^{-\frac{1}{2}|\mathbf{k}|^{2} \sigma^{2}}$ in the Fourier space. Thus, setting the Gaussian to $2 \cdot 10^{-16}$ at $|\mathbf{k}|_{\infty}=P$ with $P$ being the side-length of the bounded computational box $B=\{\mathbf{k}|| \mathbf{k} \mid \leq P\}$ in the Fourier space, we can choose $\sigma=6 / P$, a constant that is independent of the density function $\rho$.

For the convenience of the readers, we summarize the algorithm to evaluate the Poisson potential $u(\mathbf{x})$ in $2 \mathrm{D}$ in Algorithm 1.

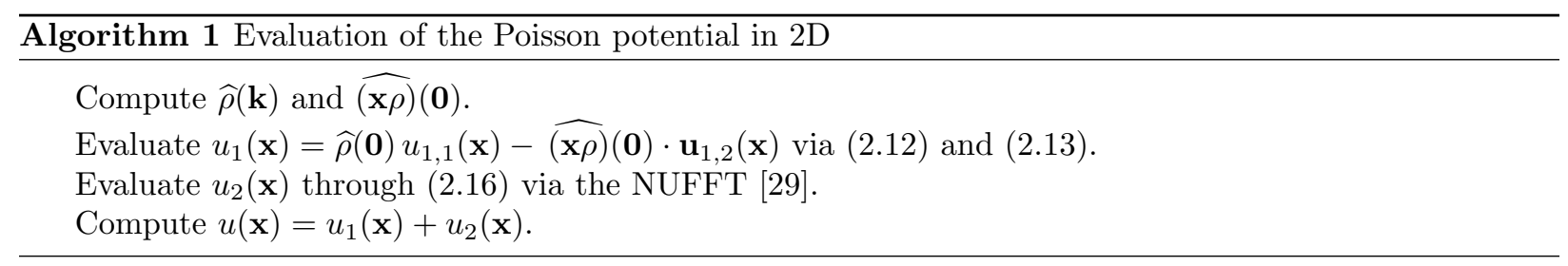

Similarly, for the $1 \mathrm{D}$ case, i.e., $U_{\text {Lap }}(x)=-\frac{1}{2}|x|$, we introduce the auxiliary functions

$$
G(x)=\frac{1}{\sqrt{2 \pi} \sigma} e^{-\frac{x^{2}}{2 \sigma^{2}}}, \quad G_{1}(x)=\widehat{\rho}(0) G(x)-\widehat{(x \rho)}(0) G^{\prime}(x), \quad x \in \mathbb{R},
$$


and function $u_{1}(x)$ which satisfies the 1D Poisson equation with the far-field condition

$$
-u_{1}^{\prime \prime}(x)=G_{1}(x), \quad x \in \mathbb{R}, \quad \lim _{x \rightarrow \pm \infty}\left[u_{1}(x)+\frac{1}{2}(\widehat{\rho}(0)|x| \mp \widehat{(x \rho)}(0))\right]=0 .
$$

Solving the above problem via the convolution, we have

$$
u_{1}(x)=\left(U_{\text {Lap }} * G_{1}\right)(x)=\widehat{\rho}(0) u_{1,1}(x)-\widehat{(x \rho)}(0) u_{1,2}(x), \quad x \in \mathbb{R},
$$

where

$$
\begin{aligned}
& u_{1,1}(x)=\left(U_{\text {Lap }} * G\right)(x)=-\frac{\sigma}{\sqrt{2 \pi}} e^{-\frac{x^{2}}{2 \sigma^{2}}}-\frac{1}{2} x \operatorname{Erf}\left(\frac{x}{\sqrt{2} \sigma}\right), \\
& u_{1,2}(x)=u_{1,1}^{\prime}(x)=-\frac{1}{2} \operatorname{Erf}\left(\frac{x}{\sqrt{2} \sigma}\right), \quad x \in \mathbb{R} .
\end{aligned}
$$

Here, $\operatorname{Erf}(x)=\frac{2}{\sqrt{\pi}} \int_{0}^{x} e^{-t^{2}} d t$ for $x \in \mathbb{R}$ is the error function. Combining (2.1) and (2.19), we solve the remaining function $u_{2}(x)=u(x)-u_{1}(x)$ via the Fourier integral:

$$
\begin{aligned}
u_{2}(x) & =\left(U_{\text {Lap }} *\left(\rho-G_{1}\right)\right)(x)=\frac{1}{2 \pi} \int_{\mathbb{R}} \frac{\widehat{\rho}(k)-\widehat{G_{1}}(k)}{k^{2}} e^{i k x} d k \\
& =\frac{1}{2 \pi} \int_{\mathbb{R}} W(k) e^{i k x} d k \approx \frac{1}{2 \pi} \int_{-P}^{P} W(k) e^{i k x} d k, \quad x \in \Omega \subset \mathbb{R},
\end{aligned}
$$

where

$$
W(k)=\left\{\begin{array}{ll}
\frac{\widehat{\rho}(k)-\widehat{G_{1}}(k)}{k^{2}}=\frac{\widehat{\rho}(k)-\left(\widehat{\rho}(0)+k(\widehat{\rho})^{\prime}(0)\right) e^{-\frac{1}{2} k^{2} \sigma^{2}}}{k^{2}}, & k \neq 0, \\
-\frac{1}{2} \widehat{\left(x^{2} \rho\right)}(0)+\frac{\sigma^{2}}{2} \widehat{\rho}(0), & k=0,
\end{array} \quad k \in \mathbb{R} .\right.
$$

Note that the integrand $W(k)$ is smooth at the origin $k=0$ in the Fourier space, therefore $u_{2}(x)$ can be computed by the regular FFT method. The choice of the parameter $\sigma$ is similar as the one in the 2D case.

We remark that the 1D Poisson potential has also been dealt with successfully in [41] by plugging the Fourier spectral approximation of the density obtained on a finite interval into the convolution formula (1.2). The method proposed there is an alternative good choice.

\subsection{Confined Coulomb interactions}

When $U(\mathbf{x})$ reads as (1.12), there is no equivalent PDE formulation for the nonlocal potential $u(\mathbf{x})$. When $d=2$, noticing that

$$
\widehat{U}_{\text {Con }}^{\varepsilon}(\mathbf{k}) \approx\left\{\begin{array}{cl}
\frac{1}{|\mathbf{k}|}, & |\mathbf{k}| \rightarrow 0, \\
\frac{\sqrt{2}}{\sqrt{\pi} \varepsilon|\mathbf{k}|^{2}}, & |\mathbf{k}| \rightarrow \infty,
\end{array} \quad \mathbf{k} \in \mathbb{R}^{2},\right.
$$

we can immediately adapt the NUFFT-based solver [29] as follows:

$$
\begin{aligned}
u(\mathbf{x}) & =\frac{1}{(2 \pi)^{2}} \int_{\mathbb{R}^{2}} e^{i \mathbf{k} \cdot \mathbf{x}} \widehat{U}_{\mathrm{Con}}^{\varepsilon}(\mathbf{k}) \widehat{\rho}(\mathbf{k}) d \mathbf{k} \approx \frac{1}{(2 \pi)^{2}} \int_{|\mathbf{k}| \leq P} e^{i \mathbf{k} \cdot \mathbf{x}} \widehat{U}_{\mathrm{Con}}^{\varepsilon}(\mathbf{k}) \widehat{\rho}(\mathbf{k}) d \mathbf{k} \\
& =\frac{1}{(2 \pi)^{2}} \int_{0}^{P} \int_{0}^{2 \pi} e^{i \mathbf{k} \cdot \mathbf{x}} W_{1}(\mathbf{k}) \widehat{\rho}(\mathbf{k}) d|\mathbf{k}| d \theta, \quad \mathbf{x} \in \Omega \subset \mathbb{R}^{2},
\end{aligned}
$$

where

$$
W_{1}(\mathbf{k})=|\mathbf{k}| \widehat{U}_{\text {Con }}^{\varepsilon}(\mathbf{k})=\frac{2}{\pi} \int_{0}^{\infty} \frac{|\mathbf{k}| e^{-\frac{\varepsilon^{2} s^{2}}{2}}}{|\mathbf{k}|^{2}+s^{2}} d s=\left\{\begin{array}{ll}
\frac{2}{\pi} \int_{0}^{\infty} \frac{e^{-\varepsilon^{2} \mid \mathbf{k} \mathbf{k}^{2} s^{2} / 2}}{1+s^{2}} d s, & \mathbf{k} \neq \mathbf{0}, \\
1, & \mathbf{k}=\mathbf{0},
\end{array} \quad \mathbf{k} \in \mathbb{R}^{2} .\right.
$$


The integral in (2.28) can be evaluated very accurately via the standard quadrature, such as the GaussKronrod quadrature.

Similarly, when $d=1$ we have

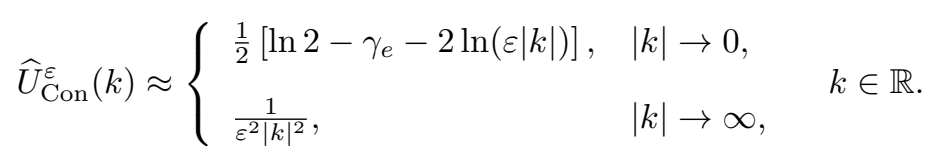

Thus

$$
\begin{aligned}
u(x) & =\frac{1}{2 \pi} \int_{\mathbb{R}} e^{i k x} \widehat{U}_{\mathrm{Con}}^{\varepsilon}(k) \widehat{\rho}(k) d k=-\frac{1}{2 \pi} \int_{\mathbb{R}} e^{i k x} k\left[\partial_{k}\left(\widehat{U}_{\mathrm{Con}}^{\varepsilon}(k) \widehat{\rho}(k)\right)+i x \widehat{U}_{\mathrm{Con}}^{\varepsilon}(k) \widehat{\rho}(k)\right] d k \\
& =-\frac{1}{2 \pi} \int_{\mathbb{R}} e^{i k x}\left[k \partial_{k} \widehat{U}_{\mathrm{Con}}^{\varepsilon}(k) \widehat{\rho}(k)-i k \widehat{U}_{\mathrm{Con}}^{\varepsilon}(k) \widehat{(x \rho)}(k)+i x k \widehat{U}_{\mathrm{Con}}^{\varepsilon}(k) \widehat{\rho}(k)\right] d k \\
& =\frac{1}{2 \pi} \int_{\mathbb{R}} e^{i k x}\left[W_{2}(k) \widehat{\rho}(k)+i W_{3}(k) \widehat{(x \rho)}(k)\right] d k-\frac{i x}{2 \pi} \int_{\mathbb{R}} e^{i k x} W_{3}(k) \widehat{\rho}(k) d k \\
& \approx \frac{1}{2 \pi} \int_{-P}^{P} e^{i k x}\left[W_{2}(k) \widehat{\rho}(k)+i W_{3}(k) \widehat{(x \rho)}(k)\right] d k-\frac{i x}{2 \pi} \int_{-P}^{P} e^{i k x} W_{3}(k) \widehat{\rho}(k) d k, x \in[-L, L] .
\end{aligned}
$$

Here

$$
\begin{aligned}
& W_{2}(k)=-k \partial_{k} \widehat{U}_{\text {Con }}^{\varepsilon}(k)=\int_{0}^{\infty} \frac{k^{2} e^{-\varepsilon^{2} s / 2}}{\left(k^{2}+s\right)^{2}} d s=\left\{\begin{array}{ll}
\int_{0}^{\infty} \frac{e^{-\varepsilon^{2} k^{2} s / 2}}{(1+s)^{2}} d s, & k \neq 0, \\
1, & k=0,
\end{array} \quad k \in \mathbb{R},\right. \\
& W_{3}(k)=k \widehat{U}_{\text {Con }}^{\varepsilon}(k)=\int_{0}^{\infty} \frac{k e^{-\varepsilon^{2} s / 2}}{2\left(k^{2}+s\right)} d s=\left\{\begin{array}{ll}
\int_{0}^{\infty} \frac{k e^{-\varepsilon^{2} k^{2} s / 2}}{2(1+s)} d s, & k \neq 0, \\
0, & k=0,
\end{array} \quad k \in \mathbb{R} .\right.
\end{aligned}
$$

The integrals in (2.31)-(2.32) can be discretized very accurately via the standard quadrature, and the integrals in (2.30) can be evaluated via the regular FFT.

Remark 2.1. If $\rho(\mathbf{x})$ is spherically/radially symmetric and $U(\mathbf{x})$ reads as (1.9) in $3 D / 2 D$, then the corresponding nonlocal interaction $u(\mathbf{x})$ in (2.1) is also spherically/radially symmetric. In addition, it satisfies the following second-order $O D E$

$$
\begin{aligned}
& -\frac{1}{r^{d-1}} \partial_{r}\left(r^{d-1} \partial_{r} u(r)\right)=\rho(r), \quad 0<r<\infty, \\
& \partial_{r} u(0)=0, \quad u(r) \rightarrow\left\{\begin{array}{cc}
0, & d=3, \\
-C_{0} \ln r, & d=2,
\end{array}\right.
\end{aligned}
$$

where $r=|\mathbf{x}|$ and $C_{0}=\int_{0}^{\infty} \rho(r) r d r$. Moreover, if $\rho(r)$ has a compact support or decays exponentially fast when $r \rightarrow \infty$, the above problem can be further re-formulated or approximated by [28, 35]

$$
\begin{aligned}
& -\frac{1}{r^{d-1}} \partial_{r}\left(r^{d-1} \partial_{r} u(r)\right)=\rho(r), \quad 0<r<L, \quad d=3,2, \\
& \partial_{r} u(0)=0, \quad \partial_{r} u(L)= \begin{cases}-\frac{u(L)}{L}, & d=3, \\
\frac{u(L)}{L \ln L}, & d=2,\end{cases}
\end{aligned}
$$

where $L>0$ is large enough such that $\operatorname{supp}(\rho) \subset[0, L]$ or the truncation error in $\rho$ outside $[0, L]$ is negligible. This two-point boundary value problem can be solved by the finite difference (FDM) or finite element (FEM) or spectral method. Comparing to computing the original convolution or solving the corresponding Poisson equation in 3D/2D, the memory and/or computational cost are significantly reduced. 


\subsection{Numerical comparisons}

In this subsection, we will demonstrate the efficiency and accuracy of the NUFFT based method for nonlocal interaction evaluation and compare it with other existing methods such as the FFT based algorithm [11], the DST based one [17, 41] and the finite difference method via (2.35)-(2.36) [35]. To this end, we denote $\Omega$ as the computational domain and choose $h_{x}=h_{y}=h_{z}=h$ in 3D or $h_{x}=h_{y}=h$ in 2D unless stated otherwise. We denote $\Omega_{h}$ as the partition of $\Omega$ with mesh size $h$ and $u_{h}(\mathbf{x})$ as the numerical solution obtained by a numerical method on domain $\Omega_{h}$. To show the comparison, we adopt the error function

$$
e_{h}:=\frac{\left\|u-u_{h}\right\|_{l^{\infty}}}{\|u\|_{l^{\infty}}}=\frac{\max _{\mathbf{x} \in \Omega_{h}}\left|u(\mathbf{x})-u_{h}(\mathbf{x})\right|}{\max _{\mathbf{x} \in \Omega_{h}}|u(\mathbf{x})|} .
$$

Example 2.1: $3 D$ Coulomb interaction. Here $d=3$ and $U(\mathbf{x})=U_{\text {Cou }}(\mathbf{x})$, we take $\rho(\mathbf{x}):=e^{-\left(x^{2}+y^{2}+\gamma^{2} z^{2}\right) / \delta^{2}}$ with $\delta>0$ and $\gamma \geq 1$. The 3D Coulomb interaction can be computed analytically as

$$
u(\mathbf{x})=\left\{\begin{array}{ll}
\frac{\delta^{3} \sqrt{\pi}}{4|\mathbf{x}|} \operatorname{Erf}\left(\frac{|\mathbf{x}|}{\delta}\right), & \gamma=1, \\
\frac{\delta^{2}}{4 \gamma} \int_{0}^{\infty} \frac{e^{-\frac{x^{2}+y^{2}}{\delta^{2}(t+1)}} e^{-\frac{z^{2}}{\delta^{2}\left(t+\gamma^{-2}\right)}}}{(t+1) \sqrt{t+\gamma^{-2}}} d t, & \gamma \neq 1,
\end{array} \quad \mathbf{x} \in \mathbb{R}^{3} .\right.
$$

The 3D Coulomb interaction $u(\mathbf{x})$ is computed numerically via the NUFFT, DST and FFT methods on a bounded computational domain $\Omega=[-L, L]^{2} \times[-L / \gamma, L / \gamma]$ with mesh size $h$. Table 1 shows the errors $e_{h}$ via the NUFFT, DST and FFT methods with $\gamma=1, \delta=1.1$ for different mesh size $h$ and $L$. Figure 2 depicts the error of the Coulomb interaction along the $x$-axis, which is defined as $\delta_{h}(x):=\left|u(x, 0,0)-u_{h}(x, 0,0)\right|$, obtained via the NUFFT and DST methods with $\gamma=1, \delta=1.1$ for different mesh size $h$ and $L$. In addition, Table 2 shows the errors $e_{h}$ via the NUFFT, DST and FFT methods with $\delta=2$ and $L=8, h=1 / 4$ for different $\gamma$. Here $h$ denote $h_{x}=h_{y}$ and we choose $h_{z}=h / \gamma$.

Table 1: Errors for the evaluation of the 3D Coulomb interaction by different methods for different $h$ and $L$.

\begin{tabular}{lrllll}
\hline NUFFT & $h=2$ & $h=1$ & $h=1 / 2$ & $h=1 / 4$ & $h=1 / 8$ \\
\hline$L=4$ & $4.191 \mathrm{E}-01$ & $2.696 \mathrm{E}-03$ & $6.634 \mathrm{E}-07$ & $4.599 \mathrm{E}-07$ & $3.688 \mathrm{E}-07$ \\
$L=8$ & $4.111 \mathrm{E}-01$ & $2.817 \mathrm{E}-03$ & $1.667 \mathrm{E}-08$ & $2.367 \mathrm{E}-14$ & $2.404 \mathrm{E}-14$ \\
$L=16$ & $4.127 \mathrm{E}-01$ & $2.848 \mathrm{E}-03$ & $1.732 \mathrm{E}-08$ & $1.420 \mathrm{E}-14$ & $1.334 \mathrm{E}-14$ \\
\hline$\overline{\text { DST }}$ & $h=2$ & $h=1$ & $h=1 / 2$ & $h=1 / 4$ & $h=1 / 8$ \\
\hline$L=4$ & $2.437 \mathrm{E}-01$ & $2.437 \mathrm{E}-01$ & $2.437 \mathrm{E}-01$ & $2.437 \mathrm{E}-01$ & $2.437 \mathrm{E}-01$ \\
$L=8$ & $2.754 \mathrm{E}-01$ & $1.219 \mathrm{E}-01$ & $1.219 \mathrm{E}-01$ & $1.219 \mathrm{E}-01$ & $1.219 \mathrm{E}-01$ \\
$L=16$ & $3.433 \mathrm{E}-01$ & $6.093 \mathrm{E}-02$ & $6.093 \mathrm{E}-02$ & $6.093 \mathrm{E}-02$ & $6.093 \mathrm{E}-02$ \\
$L=32$ & $3.780 \mathrm{E}-01$ & $3.046 \mathrm{E}-02$ & $3.046 \mathrm{E}-02$ & $3.046 \mathrm{E}-02$ & $3.046 \mathrm{E}-02$ \\
$L=64$ & $3.956 \mathrm{E}-01$ & $1.523 \mathrm{E}-02$ & $1.523 \mathrm{E}-02$ & $1.523 \mathrm{E}-02$ & $1.523 \mathrm{E}-02$ \\
\hline \hline FFT & $h=2$ & $h=1$ & $h=1 / 2$ & $h=1 / 4$ & $h=1 / 8$ \\
\hline$L=4$ & $3.032 \mathrm{E}-01$ & $3.363 \mathrm{E}-01$ & $3.385 \mathrm{E}-01$ & $3.385 \mathrm{E}-01$ & $3.385 \mathrm{E}-01$ \\
$L=8$ & $1.744 \mathrm{E}-01$ & $1.712 \mathrm{E}-01$ & $1.720 \mathrm{E}-01$ & $1.720 \mathrm{E}-01$ & $1.720 \mathrm{E}-01$ \\
$L=16$ & $2.958 \mathrm{E}-01$ & $8.666 \mathrm{E}-02$ & $8.632 \mathrm{E}-02$ & $8.632 \mathrm{E}-02$ & $8.632 \mathrm{E}-02$ \\
$L=32$ & $3.550 \mathrm{E}-01$ & $4.372 \mathrm{E}-02$ & $4.320 \mathrm{E}-02$ & $4.320 \mathrm{E}-02$ & $4.320 \mathrm{E}-02$ \\
$L=64$ & $3.843 \mathrm{E}-01$ & $2.214 \mathrm{E}-02$ & $2.161 \mathrm{E}-02$ & $2.161 \mathrm{E}-02$ & $2.161 \mathrm{E}-02$ \\
\hline
\end{tabular}

From Tables 1-2 and Figure 2, we can observe clearly that : (i) The errors are saturated in the DST and FFT methods as mesh size $h$ tends smaller and the saturated accuracies decrease linearly with respect to the box size $L$; (ii) The NUFFT method is spectrally accurate and it essentially does not depend on the domain, 
Table 2: Errors for the evaluation of the 3D Coulomb interaction by different methods with $\delta=2$ and $L=8, h=1 / 4$ for different $\gamma$.

\begin{tabular}{lrlll}
\hline & $\gamma=1$ & $\gamma=2$ & $\gamma=4$ & $\gamma=8$ \\
\hline NUFFT & $2.164 \mathrm{E}-14$ & $2.134 \mathrm{E}-14$ & $2.044 \mathrm{E}-14$ & $2.005 \mathrm{E}-14$ \\
\hline DST & 0.146 & 0.441 & 1.559 & 3.782 \\
\hline FFT & 0.208 & 0.310 & 1.327 & 3.349 \\
\hline
\end{tabular}
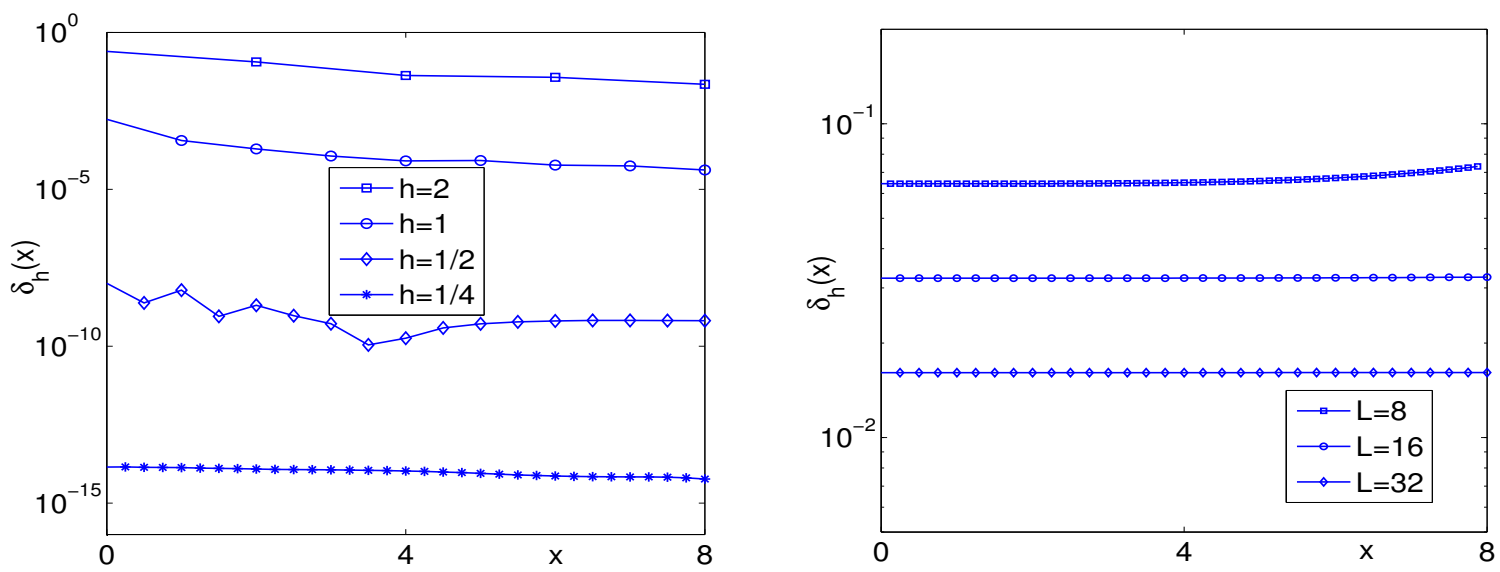

Figure 2: Errors of $\delta_{h}(x)=\left|u(x, 0,0)-u_{h}(x, 0,0)\right|$ for the evaluation of the Coulomb interaction in 3D via the NUFFT method with $L=8$ for different mesh size $h$ (left) and via the DST method with mesh size $h=1 / 4$ for different $L$ (right).

which implies that a very large bounded computational domain is not necessary in practical computations when the NUFFT method is used; (iii) The NUFFT is capable of dealing with anisotropic densities, which is quite useful in numerical simulation of BEC with strong confinement, while the errors by the DST and FFT methods increase dramatically with strongly anisotropic densities (cf. Tab. 2).

Example 2.2: $2 D$ Coulomb interaction. Here $d=2$ and $U(\mathbf{x})=U_{\text {Cou }}(\mathbf{x})$, we take $\rho(\mathbf{x}):=e^{-\left(x^{2}+\gamma^{2} y^{2}\right) / \delta^{2}}$ with $\delta>0$ and $\gamma \geq 1$. The $2 \mathrm{D}$ Coulomb interaction can be obtained analytically as

$$
u(\mathbf{x})=\left\{\begin{array}{ll}
\frac{\sqrt{\pi} \delta}{2} \mathrm{I}_{0}\left(\frac{|\mathbf{x}|^{2}}{2 \delta^{2}}\right) e^{-\frac{|\mathbf{x}|^{2}}{2 \delta^{2}}}, & \gamma=1, \\
\frac{\delta}{\gamma \sqrt{\pi}} \int_{0}^{\infty} \frac{e^{-\frac{x^{2}}{\delta^{2}\left(t^{2}+1\right)}} e^{-\frac{y^{2}}{\delta^{2}\left(t^{2}+\gamma^{-2}\right)}}}{\sqrt{t^{2}+1} \sqrt{t^{2}+\gamma^{-2}}} d t, & \gamma \neq 1,
\end{array} \quad \mathbf{x} \in \mathbb{R}^{2},\right.
$$

where $\mathrm{I}_{0}$ is the modified Bessel function of order zero [1]. To numerically compute the integral in (2.39), we first split it into two integrals and reformulate the one with infinite interval into some equivalent integral with finite interval by a simple change of variable. We then apply the Gauss-Kronrod quadrature to each with fine accuracy control so as to achieve accurate reference solutions.

The 2D Coulomb interaction $u(\mathbf{x})$ is computed numerically via the NUFFT, DST and FFT methods on a bounded computational domain $\Omega=[-L, L] \times[-L / \gamma, L / \gamma]$ with mesh size $h$. Table 3 shows the errors $e_{h}$ via the NUFFT, DST and FFT methods with $\delta=\sqrt{1.2}$ and $\gamma=1$ under different mesh size $h$ and $L$. In addition, Table 4 shows the errors $e_{h}$ via the NUFFT, DST and FFT methods with $\delta=2, L=12$ and $h=1 / 8$ for different $\gamma$.Here $h$ denote $h_{x}$ and we choose $h_{y}=h / \gamma$.

From Tables 3-4, we can conclude that: (i) The errors obtained by the DST and FFT methods reach a saturation accuracy on any fixed domain and we can observe a first order convergence in the saturated 
Table 3: Errors for the evaluation of the 2D Coulomb interaction by different methods for different $h$ and $L$.

\begin{tabular}{llllll}
\hline NUFFT & $h=2$ & $h=1$ & $h=1 / 2$ & $h=1 / 4$ & $h=1 / 8$ \\
\hline$L=4$ & 1.837 & $5.540 \mathrm{E}-02$ & $4.289 \mathrm{E}-07$ & $3.383 \mathrm{E}-07$ & $2.937 \mathrm{E}-07$ \\
$L=8$ & $4.457 \mathrm{E}-01$ & $2.373 \mathrm{E}-03$ & $2.714 \mathrm{E}-08$ & $3.202 \mathrm{E}-15$ & $3.431 \mathrm{E}-15$ \\
$L=16$ & $2.084 \mathrm{E}-01$ & $2.385 \mathrm{E}-03$ & $2.761 \mathrm{E}-08$ & $2.745 \mathrm{E}-15$ & $2.859 \mathrm{E}-15$ \\
\hline$\overline{\text { DST }}$ & $h=2$ & $h=1$ & $h=1 / 2$ & $h=1 / 4$ & $h=1 / 8$ \\
\hline$L=4$ & $1.577 \mathrm{E}-01$ & $1.577 \mathrm{E}-01$ & $1.577 \mathrm{E}-01$ & $1.577 \mathrm{E}-01$ & $1.577 \mathrm{E}-01$ \\
$L=8$ & $1.348 \mathrm{E}-01$ & $7.762 \mathrm{E}-02$ & $7.762 \mathrm{E}-02$ & $7.762 \mathrm{E}-02$ & $7.762 \mathrm{E}-02$ \\
$L=16$ & $1.711 \mathrm{E}-01$ & $3.867 \mathrm{E}-02$ & $3.867 \mathrm{E}-02$ & $3.867 \mathrm{E}-02$ & $3.867 \mathrm{E}-02$ \\
$L=32$ & $1.897 \mathrm{E}-01$ & $1.932 \mathrm{E}-02$ & $1.932 \mathrm{E}-02$ & $1.932 \mathrm{E}-02$ & $1.932 \mathrm{E}-02$ \\
$L=64$ & $1.991 \mathrm{E}-01$ & $9.658 \mathrm{E}-03$ & $9.658 \mathrm{E}-03$ & $9.658 \mathrm{E}-03$ & $9.658 \mathrm{E}-03$ \\
\hline$\overline{\text { FFT }}$ & $h=2$ & $h=1$ & $h=1 / 2$ & $h=1 / 4$ & $h=1 / 8$ \\
\hline$L=4$ & $2.855 \mathrm{E}-01$ & $2.961 \mathrm{E}-01$ & $2.980 \mathrm{E}-01$ & $2.980 \mathrm{E}-01$ & $2.980 \mathrm{E}-01$ \\
$L=8$ & $1.553 \mathrm{E}-01$ & $1.503 \mathrm{E}-01$ & $1.502 \mathrm{E}-01$ & $1.502 \mathrm{E}-01$ & $1.502 \mathrm{E}-01$ \\
$L=16$ & $1.157 \mathrm{E}-01$ & $7.596 \mathrm{E}-02$ & $7.528 \mathrm{E}-02$ & $7.528 \mathrm{E}-02$ & $7.528 \mathrm{E}-02$ \\
$L=32$ & $1.624 \mathrm{E}-01$ & $3.843 \mathrm{E}-02$ & $3.766 \mathrm{E}-02$ & $3.766 \mathrm{E}-02$ & $3.766 \mathrm{E}-02$ \\
$L=64$ & $1.856 \mathrm{E}-01$ & $1.961 \mathrm{E}-02$ & $1.883 \mathrm{E}-02$ & $1.883 \mathrm{E}-02$ & $1.883 \mathrm{E}-02$ \\
\hline
\end{tabular}

Table 4: Errors for the evaluation of the 2D Coulomb interaction by different methods with $L=12, h=1 / 8$ for different $\gamma$.

\begin{tabular}{lrlll}
\hline & $\gamma=1$ & $\gamma=2$ & $\gamma=4$ & $\gamma=8$ \\
\hline NUFFT & $4.230 \mathrm{E}-14$ & $3.102 \mathrm{E}-15$ & $3.504 \mathrm{E}-15$ & $4.381 \mathrm{E}-15$ \\
\hline DST & 0.373 & 0.386 & 0.412 & 0.446 \\
\hline FFT & 0.426 & 0.425 & 0.405 & 0.344 \\
\hline
\end{tabular}

accuracy with respect to the domain size $L$. (ii) The NUFFT method is spectrally accurate and it essentially does not depend on the domain which makes it perfect for computing the whole space potential. (iii) The NUFFT is capable of dealing with anisotropic densities, while the results obtained by the DST and FFT methods are far from the exact solutions when the bounded computational domain is not large enough.

Example 2.3: $2 D$ Poisson potential. Here $d=2$ and $U(\mathbf{x})=U_{\text {Lap }}(\mathbf{x})$, we take $\rho(\mathbf{x}):=e^{-|\mathbf{x}|^{2} / \delta^{2}}=$ $e^{-r^{2} / \delta^{2}}$ with $r=|\mathbf{x}|$ and $\delta>0$. The 2D Poisson potential can be obtained analytically as

$$
u(\mathbf{x})=-\frac{\delta^{2}}{4}\left[\mathrm{E}_{1}\left(\frac{|\mathbf{x}|^{2}}{\delta^{2}}\right)+2 \ln (|\mathbf{x}|)\right], \quad \mathbf{x} \in \mathbb{R}^{2} .
$$

In this case, we choose $\delta=\sqrt{1.3}$. The $2 \mathrm{D}$ Poisson potential $u(\mathbf{x})$ is computed numerically via the NUFFT method on a bounded computational domain $\Omega=[-L, L]^{2}$ with mesh size $h$ and the FDM through the formulation (2.35)-(2.36) on the interval $[0, L]$ with mesh size $h$.

Table 5 shows the errors of the 2D Poisson potential obtained by the NUFFT solver on a square domain and the errors by the FDM solver as well as its convergence rate with respect to the mesh size $h$. In addition, to demonstrate the efficiency of the NUFFT method, Table 6 displays the computational time (CPU time in seconds) of the NUFFT solver with $L=16$ and $h=1 / 4$, where the time is measured when the algorithm is implemented in Fortran, the code is compiled by ifort 13.1.2 using the option -g, and executed on 32-bit Ubuntu Linux on a $2.90 \mathrm{GHz} \operatorname{Intel}(\mathrm{R})$ Core(TM) i7-3520M CPU with 6MB cache. 
Table 5: Errors for the evaluation of the 2D Poisson potential by different methods for different $h$ and $L$.

\begin{tabular}{lrllll}
\hline NUFFT & $h=2$ & $h=1$ & $h=1 / 2$ & $h=1 / 4$ & $h=1 / 8$ \\
\hline$L=4$ & $5.821 \mathrm{E}-01$ & $1.133 \mathrm{E}-02$ & $3.011 \mathrm{E}-06$ & $1.994 \mathrm{E}-06$ & $1.650 \mathrm{E}-06$ \\
$L=8$ & $1.685 \mathrm{E}-01$ & $6.820 \mathrm{E}-04$ & $1.754 \mathrm{E}-09$ & $4.936 \mathrm{E}-14$ & $4.857 \mathrm{E}-14$ \\
$L=16$ & $1.684 \mathrm{E}-01$ & $5.333 \mathrm{E}-04$ & $1.391 \mathrm{E}-09$ & $4.577 \mathrm{E}-14$ & $4.561 \mathrm{E}-14$ \\
\hline \hline FDM & $h=1 / 4$ & $h=1 / 8$ & $h=1 / 16$ & $h=1 / 32$ & $h=1 / 64$ \\
\hline$L=4$ & $4.646 \mathrm{E}-03$ & $1.155 \mathrm{E}-03$ & $2.910 \mathrm{E}-04$ & $7.602 \mathrm{E}-05$ & $2.246 \mathrm{E}-05$ \\
rate & - & 2.0081 & 1.9889 & 1.9365 & 1.7590 \\
$L=8$ & $4.101 \mathrm{E}-03$ & $1.019 \mathrm{E}-03$ & $2.542 \mathrm{E}-04$ & $6.353 \mathrm{E}-05$ & $1.588 \mathrm{E}-05$ \\
rate & - & 2.0093 & 2.0024 & 2.0006 & 2.0002 \\
$L=16$ & $4.052 \mathrm{E}-03$ & $1.007 \mathrm{E}-03$ & $2.512 \mathrm{E}-04$ & $6.278 \mathrm{E}-05$ & $1.569 \mathrm{E}-05$ \\
rate & - & 2.0092 & 2.0023 & 2.0006 & 2.0001 \\
\hline
\end{tabular}

Table 6: CPU time (in seconds) of the NUFFT solver for the evaluation of the 2D Poisson potential. Here $T_{\mathrm{FFT}}$ and $T_{\mathrm{NUFFT}}$ are the time for the evaluation of $I_{1}$ and $I_{2}$ in (2.3) via the FFT and NUFFT methods, respectively.

\begin{tabular}{lccc}
\hline & $T_{\mathrm{FFT}}$ & $T_{\text {NUFFT }}$ & $T_{\text {Total }}$ \\
\hline$h=1$ & 0.01 & 0.05 & 0.06 \\
$h=1 / 2$ & 0.02 & 0.08 & 0.10 \\
$h=1 / 4$ & 0.12 & 0.20 & 0.32 \\
$h=1 / 8$ & 0.60 & 0.78 & 1.38 \\
\hline
\end{tabular}

From Tables 5-6, we can see clearly that: (i) The NUFFT solver is spectrally accurate while the FDM solver is only second order accurate, and the NUFFT solver is much more accurate than the FDM solver. (ii) The errors obtained by both methods do not essentially depend on the domain size; (iii) The complexity of the NUFFT solver scales like $O(N \ln N)$ as expected, which is the same as those presented in [29].

\section{Computing the ground state}

In this section, we present an efficient and accurate numerical method for computing the ground state of (1.13) by combining NUFFT-based nonlocal interaction potential solver and the normalized gradient flow that is discretised by backward Euler Fourier pseudospectral method, and compare it with those existing numerical methods.

\subsection{A numerical method via the NUFFT}

We choose $\tau>0$ as the time step and denote $t_{n}=n \tau$ for $n=0,1,2, \ldots$ Different efficient and accurate numerical methods have been proposed in the literature for computing the ground state $[6,7,8,21,41]$. One of the most simple and popular methods is through the following gradient flow with discretized normalization (GFDN):

$$
\begin{aligned}
\partial_{t} \phi(\mathbf{x}, t) & =\left[\frac{1}{2} \Delta-V(\mathbf{x})-\beta \varphi(\mathbf{x}, t)\right] \phi(\mathbf{x}, t), \quad \mathbf{x} \in \mathbb{R}^{d}, \quad t_{n} \leq t<t_{n+1}, \\
\varphi(\mathbf{x}, t) & =\left(U *|\phi|^{2}\right)(\mathbf{x}, t), \quad \mathbf{x} \in \mathbb{R}^{d}, \quad t_{n} \leq t<t_{n+1}, \\
\phi\left(\mathbf{x}, t_{n+1}\right) & :=\phi\left(\mathbf{x}, t_{n+1}^{+}\right)=\frac{\phi\left(\mathbf{x}, t_{n+1}^{-}\right)}{\left\|\phi\left(\mathbf{x}, t_{n+1}^{-}\right)\right\|}, \quad \mathbf{x} \in \mathbb{R}^{d}, \quad n=0,1,2, \ldots
\end{aligned}
$$


with the initial data

$$
\phi(\mathbf{x}, 0)=\phi_{0}(\mathbf{x}), \quad \mathbf{x} \in \mathbb{R}^{d}, \quad \text { with } \quad\left\|\phi_{0}\right\|^{2}:=\int_{\mathbb{R}^{d}}\left|\phi_{0}(\mathbf{x})\right|^{2} d \mathbf{x}=1 .
$$

Let $\phi^{n}(\mathbf{x})$ and $\varphi^{n}(\mathbf{x})$ be the numerical approximation of $\phi\left(\mathbf{x}, t_{n}\right)$ and $\varphi\left(\mathbf{x}, t_{n}\right)$, respectively, for $n \geq 0$. The above GFDN is usually discretized in time via the backward Euler method [6, 7, 8, 21, 41]

$$
\begin{aligned}
& \frac{\phi^{(1)}(\mathbf{x})-\phi^{n}(\mathbf{x})}{\tau}=\left[\frac{1}{2} \Delta-V(\mathbf{x})-\beta \varphi^{n}(\mathbf{x})\right] \phi^{(1)}(\mathbf{x}), \quad \mathbf{x} \in \mathbb{R}^{d}, \\
& \varphi^{n}(\mathbf{x})=\left(U *\left|\phi^{n}\right|^{2}\right)(\mathbf{x}), \quad \mathbf{x} \in \mathbb{R}^{d}, \\
& \phi^{n+1}(\mathbf{x})=\frac{\phi^{(1)}(\mathbf{x})}{\left\|\phi^{(1)}(\mathbf{x})\right\|}, \quad \mathbf{x} \in \mathbb{R}^{d}, \quad n=0,1,2, \ldots .
\end{aligned}
$$

Then an efficient and accurate numerical method can be designed by: (i) truncating the above problem on a bounded computational domain $\Omega$ with periodic $\mathrm{BC}$ on $\partial \Omega$; (ii) discretizing in space via the Fourier pseudospectral method; and (iii) evaluating the nonlocal interaction $\varphi^{n}(\mathbf{x})$ in (3.6) by the algorithm via the NUFFT discussed in the previous section. When $\phi_{0}(\mathbf{x})$ is chosen as a positive function, the ground state can be obtained as $\phi_{g}(\mathbf{x})=\lim _{n \rightarrow \infty} \phi^{n}(\mathbf{x})$ for $\mathbf{x} \in \Omega$. The details are omitted here for brevity and this method is referred as the GF-NUFFT method. We remark here that $\widehat{\left|\phi^{n}\right|^{2}}(\mathbf{0})=1$ for $n \geq 0$.

For comparison, for the Coulomb interaction in $3 \mathrm{D} / 2 \mathrm{D}$, when the NUFFT solver is replaced by the standard FFT, we refer the method as GF-FFT. In addition, when (3.6) is reformulated as its equivalent PDE formulation (1.7)-(1.8) on $\Omega$ with homogeneous Dirichlet $\mathrm{BC}$ on $\partial \Omega$ and solved via the sine pseudospectral method [6, 9, 41], we refer it as GF-DST.

\subsection{Numerical comparisons}

In order to compare the GF-NUFFT method with GF-FFT and GF-DST methods for computing the ground state, we denote $\varphi_{g}(\mathbf{x})=\left(U *\left|\phi_{g}\right|^{2}\right)(\mathbf{x})$ and introduce the errors

$$
e_{\phi_{g}}^{h}:=\frac{\max _{\mathbf{x} \in \Omega^{h}}\left|\phi_{g}(\mathbf{x})-\phi_{g}^{h}(\mathbf{x})\right|}{\max _{\mathbf{x} \in \Omega^{h}}\left|\phi_{g}(\mathbf{x})\right|}, \quad e_{\varphi_{g}}^{h}:=\frac{\max _{\mathbf{x} \in \Omega^{h}}\left|\varphi_{g}(\mathbf{x})-\varphi_{g}^{h}(\mathbf{x})\right|}{\max _{\mathbf{x} \in \Omega^{h}}\left|\varphi_{g}(\mathbf{x})\right|},
$$

where $\phi_{g}^{h}$ and $\varphi_{g}^{h}$ are obtained numerically by a numerical method with mesh size $h$. Additionally, we split the energy functional into three parts

$$
E(\phi)=E_{\mathrm{kin}}(\phi)+E_{\mathrm{pot}}(\phi)+E_{\mathrm{int}}(\phi),
$$

where the kinetic energy $E_{\text {kin }}(\phi)$, the potential energy $E_{\text {pot }}(\phi)$ and the interaction energy $E_{\text {int }}(\phi)$ are defined as

$$
E_{\text {kin }}(\phi)=\frac{1}{2} \int_{\mathbb{R}^{d}}|\nabla \phi(\mathbf{x})|^{2} d \mathbf{x}, \quad E_{\mathrm{pot}}(\phi)=\int_{\mathbb{R}^{d}} V(\mathbf{x})|\phi(\mathbf{x})|^{2} d \mathbf{x}, \quad E_{\mathrm{int}}(\phi)=\frac{\beta}{2} \int_{\mathbb{R}^{d}} \varphi(\mathbf{x})|\phi(\mathbf{x})|^{2} d \mathbf{x},
$$

respectively. Moreover, the chemical potential can be reformulated as $\mu(\phi)=E(\phi)+E_{\text {int }}(\phi)$. Furthermore, if the external potential $V(\mathbf{x})$ in (1.1) was taken as the harmonic potential $[4,9,35]$, the energies of the ground state satisfy the following virial identity

$$
0=I:=2 E_{\text {kin }}\left(\phi_{g}\right)-2 E_{\text {pot }}\left(\phi_{g}\right)+ \begin{cases}E_{\text {int }}\left(\phi_{g}\right), & U=U_{\text {Cou }} \text { in } 3 \mathrm{D} / 2 \mathrm{D} \\ \frac{\beta}{4 \pi}, & U=U_{\text {Lap }} \text { in } 2 \mathrm{D}\end{cases}
$$

We denote $I^{h}$ as an approximation of $I$ when $\phi_{g}$ is replace by $\phi_{g}^{h}$ in the above equality. In our computations, the ground state $\phi_{g}^{h}$ is reached numerically when $\max _{\mathbf{x} \in \Omega^{h}} \frac{\left|\phi^{n+1}(\mathbf{x})-\phi^{n}(\mathbf{x})\right|}{\tau} \leq \varepsilon_{0}$ with $\varepsilon_{0}$ a prescribed accuracy, e.g., $\varepsilon_{0}=10^{-10}$. The initial data $\phi_{0}(\mathbf{x})$ is chosen as a Gaussian and the time step is taken as $\tau=10^{-2}$. In 
the comparisons, the reference solution $\phi_{g}(\mathbf{x})$ was obtained numerically via the GF-NUFFT method on a large enough domain $\Omega=[-8,8]^{d}$ with small enough mesh size $h=2^{d-6}$ and time step $\tau=10^{-2}$.

Example 3.1: The NLSE with the Coulomb interaction in $3 D$. We take $d=3$ and $U(\mathbf{x})=U_{\mathrm{Cou}}(\mathbf{x})$ in (1.1)-(1.2). The ground state is computed numerically on a bounded domain $\Omega=[-8,8]^{3}$. Table 7 shows the errors $e_{\phi_{g}}^{h}$ and $e_{\varphi_{g}}^{h}$ with $V(\mathbf{x})=\frac{1}{2}\left(x^{2}+y^{2}+z^{2}\right)$ in (1.1) for different numerical methods, $\beta$ and mesh size $h$. In addition, Table 8 lists the energy $E_{g}:=E\left(\phi_{g}^{h}\right)$, chemical potential $\mu_{g}:=\mu\left(\phi_{g}^{h}\right)$, kinetic energy $E_{\text {kin }}^{g}:=E_{\text {kin }}\left(\phi_{g}^{h}\right)$, potential energy $E_{\text {pot }}^{g}:=E_{\text {pot }}\left(\phi_{g}^{h}\right)$, interaction energy $E_{\text {int }}^{g}:=E_{\text {int }}\left(\phi_{g}^{h}\right)$ and $I^{h}$ with $h=1 / 8$ and $V(\mathbf{x})=\frac{1}{2}\left(x^{2}+y^{2}+4 z^{2}\right)$ in (1.1) for different $\beta$.

Table 7: Errors of the ground state for the NLSE with the 3D Coulomb interaction for different methods and mesh size $h$.

\begin{tabular}{|c|c|c|c|c|c|}
\hline \multicolumn{2}{|c|}{ GF-NUFFT } & $h=2$ & $h=1$ & $h=1 / 2$ & $h=1 / 4$ \\
\hline${ }^{h}$ & $\beta=-5$ & $5.362 \mathrm{E}-02$ & $1.954 \mathrm{E}-04$ & $2.201 \mathrm{E}-07$ & $4.643 \mathrm{E}-11$ \\
\hline$e_{\phi_{g}}$ & $\beta=5$ & $1.512 \mathrm{E}-01$ & 4.712E-04 & $4.026 \mathrm{E}-08$ & $1.141 \mathrm{E}-10$ \\
\hline$h$ & $\beta=-5$ & $2.532 \mathrm{E}-01$ & $3.769 \mathrm{E}-03$ & $8.153 \mathrm{E}-07$ & $7.035 \mathrm{E}-11$ \\
\hline$e_{\varphi_{g}}$ & $\beta=5$ & $2.682 \mathrm{E}-01$ & 7.061E-04 & $1.225 \mathrm{E}-07$ & $8.048 \mathrm{E}-11$ \\
\hline \multicolumn{2}{|c|}{ GF-DST } & $h=2$ & $\bar{h} h=1$ & $h=1 / 2$ & $h=1 / 4$ \\
\hline \multirow{2}{*}{$e_{\phi_{g}}^{h}$} & $\beta=-5$ & $2.319 \mathrm{E}-01$ & $9.439 \mathrm{E}-03$ & $1.637 \mathrm{E}-06$ & $6.309 \mathrm{E}-07$ \\
\hline & $\beta=5$ & $1.659 \mathrm{E}-01$ & $9.469 \mathrm{E}-04$ & $8.306 \mathrm{E}-07$ & $8.531 \mathrm{E}-07$ \\
\hline \multirow{2}{*}{$\overline{e_{\varphi_{g}}^{h}}$} & $\beta=-5$ & $7.297 \mathrm{E}-02$ & $9.551 \mathrm{E}-02$ & $9.945 \mathrm{E}-02$ & $1.027 \mathrm{E}-01$ \\
\hline & $\beta=5$ & 7.809E-02 & $1.016 \mathrm{E}-01$ & $1.057 \mathrm{E}-01$ & $1.091 \mathrm{E}-01$ \\
\hline
\end{tabular}

Table 8: Different energies of the ground state and $I^{h}$ for the NLSE with the 3D Coulomb interaction for different $\beta$.

\begin{tabular}{llccccc}
\hline$\beta$ & $E_{g}$ & $\mu_{g}$ & $E_{\text {kin }}^{g}$ & $E_{\text {pot }}^{g}$ & $E_{\text {int }}^{g}$ & $I^{h}$ \\
\hline-10 & 1.6370 & 1.2630 & 1.0990 & $9.1197 \mathrm{E}-01$ & $-3.7401 \mathrm{E}-01$ & $-3.39 \mathrm{E}-10$ \\
-5 & 1.8212 & 1.6397 & 1.0467 & $9.5594 \mathrm{E}-01$ & $-1.8147 \mathrm{E}-01$ & $-3.63 \mathrm{E}-10$ \\
-1 & 1.9646 & 1.9292 & 1.0089 & $9.9118 \mathrm{E}-01$ & $-3.5462 \mathrm{E}-02$ & $-3.87 \mathrm{E}-10$ \\
1 & 2.0351 & 2.0702 & $9.9128 \mathrm{E}-01$ & 1.0088 & $3.5064 \mathrm{E}-02$ & $-3.86 \mathrm{E}-10$ \\
5 & 2.1739 & 2.3454 & $9.5831 \mathrm{E}-01$ & 1.0441 & $1.7151 \mathrm{E}-01$ & $-4.30 \mathrm{E}-10$ \\
10 & 2.3431 & 2.6772 & $9.2101 \mathrm{E}-01$ & 1.0880 & $3.3408 \mathrm{E}-01$ & $-1.16 \mathrm{E}-10$ \\
\hline
\end{tabular}

Example 3.2: The NLSE with the Coulomb interaction in $2 D$. We take $d=2$ and $U(\mathbf{x})=U_{\text {Cou }}(\mathbf{x})$ in (1.1)-(1.2). The ground state is computed numerically on a bounded domain $\Omega=[-L, L]^{2}$ with different mesh size $h$. Table 9 shows the errors $e_{\phi_{g}}^{h}$ and $e_{\varphi_{g}}^{h}$ with $V(\mathbf{x})=\frac{1}{2}\left(x^{2}+4 y^{2}\right)$ for different numerical methods, $\beta$ and mesh size $h$ on $[-L, L]^{2}$. In addition, Table 10 lists the energy $E_{g}:=E\left(\phi_{g}^{h}\right)$, chemical potential $\mu_{g}:=\mu\left(\phi_{g}^{h}\right)$, kinetic energy $E_{\text {kin }}^{g}:=E_{\text {kin }}\left(\phi_{g}^{h}\right)$, potential energy $E_{\text {pot }}^{g}:=E_{\text {pot }}\left(\phi_{g}^{h}\right)$, interaction energy $E_{\text {int }}^{g}:=E_{\text {int }}\left(\phi_{g}^{h}\right)$ and $I^{h}$ with $h=1 / 8$ and $V(\mathbf{x})=\frac{1}{2}\left(x^{2}+4 y^{2}\right)$ on $[-8,8]^{2}$ for different $\beta$.

Example 3.3: The NLSE with the Poisson potential in $2 D$. We take $d=2$ and $U(\mathbf{x})=U_{\text {Lap }}(\mathbf{x})$ in (1.1)(1.2). The ground state is computed numerically on a bounded domain $\Omega=[-8,8]^{2}$ with different mesh size $h$. Table 11 shows the errors $e_{\phi_{g}}^{h}$ and $e_{\varphi_{g}}^{h}$ with $V(\mathbf{x})=\frac{1}{2}\left(x^{2}+4 y^{2}\right)$ in (1.1) for different numerical methods, $\beta$ and mesh size $h$. In addition, Table 12 lists the energy $E_{g}:=E\left(\phi_{g}^{h}\right)$, chemical potential $\mu_{g}:=\mu\left(\phi_{g}^{h}\right)$, 
Table 9: Errors of the ground state for the NLSE with 2D Coulomb interaction on $[-L, L]^{2}$ with mesh size $h$.

\begin{tabular}{l|l|llll}
\hline \multicolumn{2}{c|}{ GF-NUFFT $(L=8)$} & $h=1$ & $h=1 / 2$ & $h=1 / 4$ & $h=1 / 8$ \\
\hline$e_{\phi_{g}}^{h}$ & $\beta=-5$ & $4.620 \mathrm{E}-02$ & $1.058 \mathrm{E}-03$ & $5.570 \mathrm{E}-08$ & $3.968 \mathrm{E}-15$ \\
\hline$e_{\varphi_{g}}^{h}$ & $\beta=5$ & $7.034 \mathrm{E}-03$ & $2.365 \mathrm{E}-05$ & $2.632 \mathrm{E}-10$ & $2.074 \mathrm{E}-15$ \\
\hline \hline \multicolumn{2}{c|}{ GF-DST $(L=8)$} & $1.025 \mathrm{E}-01$ & $1.402 \mathrm{E}-03$ & $8.244 \mathrm{E}-08$ & $4.445 \mathrm{E}-15$ \\
\hline$e_{\phi_{g}}^{h}$ & $\beta=-5$ & $1.263 \mathrm{E}-02$ & $3.239 \mathrm{E}-05$ & $3.161 \mathrm{E}-10$ & $1.703 \mathrm{E}-15$ \\
\hline$e_{\varphi_{g}}^{h}$ & $\beta=5$ & $h=1$ & $h=1 / 2$ & $h=1 / 4$ & $h=1 / 8$ \\
\hline \hline \multicolumn{2}{r|}{ GF-DST $(h=1 / 8)$} & $4.823 \mathrm{E}-02$ & $1.112 \mathrm{E}-03$ & $3.139 \mathrm{E}-05$ & $3.133 \mathrm{E}-05$ \\
\hline$e_{\phi_{g}}^{h}$ & $\beta=-5$ & $8.183 \mathrm{E}-03$ & $7.245 \mathrm{E}-05$ & $5.317 \mathrm{E}-05$ & $5.381 \mathrm{E}-05$ \\
\hline$e_{\varphi_{g}}^{h}$ & $\beta=-5$ & $6.613 \mathrm{E}-02$ & $5.159 \mathrm{E}-02$ & $5.159 \mathrm{E}-02$ & $5.159 \mathrm{E}-02$ \\
& $\beta=-5$ & $6.840 \mathrm{E}-02$ & $6.840 \mathrm{E}-02$ & $6.840 \mathrm{E}-02$ & $6.840 \mathrm{E}-02$ \\
\hline
\end{tabular}

Table 10: Different energies of the ground state and $I^{h}$ for the NLSE with the 2D Coulomb interaction for different $\beta$.

\begin{tabular}{llccccc}
\hline$\beta$ & $E_{g}$ & $\mu_{g}$ & $E_{\text {kin }}^{g}$ & $E_{\text {pot }}^{g}$ & $E_{\text {int }}^{g}$ & $I^{h}$ \\
\hline-10 & 0.1367 & -1.4536 & 1.2611 & $4.6592 \mathrm{E}-01$ & -1.5903 & $1.89 \mathrm{E}-10$ \\
-5 & 0.8698 & 0.1933 & $9.4226 \mathrm{E}-01$ & $6.0401 \mathrm{E}-01$ & $-6.7651 \mathrm{E}-01$ & $2.37 \mathrm{E}-10$ \\
-1 & 1.3808 & 1.2600 & $7.8098 \mathrm{E}-01$ & $7.2058 \mathrm{E}-01$ & $-1.2080 \mathrm{E}-01$ & $2.60 \mathrm{E}-10$ \\
1 & 1.6163 & 1.7311 & $7.2201 \mathrm{E}-01$ & $7.7942 \mathrm{E}-01$ & $1.1483 \mathrm{E}-01$ & $-2.61 \mathrm{E}-10$ \\
5 & 2.0551 & 2.5801 & $6.3379 \mathrm{E}-01$ & $8.9629 \mathrm{E}-01$ & $5.2501 \mathrm{E}-01$ & $-2.65 \mathrm{E}-10$ \\
10 & 2.5557 & 3.5132 & $5.5977 \mathrm{E}-01$ & 1.0385 & $9.5748 \mathrm{E}-01$ & $-2.69 \mathrm{E}-10$ \\
\hline
\end{tabular}

kinetic energy $E_{\text {kin }}^{g}:=E_{\text {kin }}\left(\phi_{g}^{h}\right)$, potential energy $E_{\text {pot }}^{g}:=E_{\text {pot }}\left(\phi_{g}^{h}\right)$, interaction energy $E_{\text {int }}^{g}:=E_{\text {int }}\left(\phi_{g}^{h}\right)$ and $I^{h}$ with $h=1 / 8$ and $V(\mathbf{x})=\frac{1}{2}\left(x^{2}+4 y^{2}\right)$ in $(1.1)$ for different $\beta$.

From Tables 7-12 and additional numerical results not shown here for brevity, we can see that: (i) The GF-NUFFT method is spectrally accurate in space, while the GF-DST method has a saturation accuracy for a fixed domain; (ii) The saturation error of the GF-DST depends inversely on the domain size $L$, and it can only reach satisfactory accuracy for some large $L$; (iii) High accuracy, i.e., 9-digit accurate, is achieved by GF-NUFFT as quite expected in the energies, which, in another way, manifest the high-accuracy advantage of our NUFFT solver.

Table 11: Errors of the ground state for the NLSE with the 2D Poisson potential with mesh size $h$.

\begin{tabular}{l|l|llll}
\hline \multicolumn{2}{c|}{ GF-NUFFT } & $h=1$ & $h=1 / 2$ & $h=1 / 4$ & $h=1 / 8$ \\
\hline$e_{\phi_{g}}^{h}$ & $\beta=-5$ & $2.465 \mathrm{E}-02$ & $1.024 \mathrm{E}-04$ & $4.699 \mathrm{E}-10$ & $2.878 \mathrm{E}-15$ \\
\hline$e_{\varphi_{g}}^{h}$ & $\beta=5$ & $1.191 \mathrm{E}-02$ & $1.593 \mathrm{E}-05$ & $9.793 \mathrm{E}-12$ & $2.726 \mathrm{E}-15$ \\
\hline
\end{tabular}


Table 12: Different energies of the ground state and $I^{h}$ for the NLSE with the 2D Poisson potential for different $\beta$.

\begin{tabular}{llccccc}
\hline$\beta$ & $E_{g}$ & $\mu_{g}$ & $E_{\text {kin }}^{g}$ & $E_{\text {pot }}^{g}$ & $E_{\text {int }}^{g}$ & $I^{h}$ \\
\hline-10 & 1.3533 & 1.1432 & $9.8061 \mathrm{E}-01$ & $5.8272 \mathrm{E}-01$ & $-2.1008 \mathrm{E}-01$ & $2.44 \mathrm{E}-10$ \\
-5 & 1.4429 & 1.3691 & $8.5784 \mathrm{E}-01$ & $6.5889 \mathrm{E}-01$ & $-7.3819 \mathrm{E}-02$ & $2.54 \mathrm{E}-10$ \\
-1 & 1.4913 & 1.4819 & $7.7024 \mathrm{E}-01$ & $7.3045 \mathrm{E}-01$ & $-9.3826 \mathrm{E}-03$ & $2.59 \mathrm{E}-10$ \\
1 & 1.5073 & 1.5139 & $7.3046 \mathrm{E}-01$ & $7.7025 \mathrm{E}-01$ & $6.5762 \mathrm{E}-03$ & $-2.62 \mathrm{E}-10$ \\
5 & 1.5221 & 1.5260 & $6.5959 \mathrm{E}-01$ & $8.5854 \mathrm{E}-01$ & $3.9516 \mathrm{E}-03$ & $-2.70 \mathrm{E}-10$ \\
10 & 1.5076 & 1.4420 & $5.8770 \mathrm{E}-01$ & $9.8559 \mathrm{E}-01$ & $-6.5660 \mathrm{E}-02$ & $-2.81 \mathrm{E}-10$ \\
\hline
\end{tabular}

\section{For computing the dynamics}

In this section, we present an efficient and accurate numerical method for computing the dynamics of the NLSE with the nonlocal interaction potential (1.1)-(1.2) and the initial data (1.3) by combining the NUFFT solver for the nonlocal interaction potential evaluation and the time-splitting Fourier pseudospectral discretization, and compare it with those existing numerical methods.

\subsection{A numerical method via the NUFFT}

From time $t=t_{n}$ to $t=t_{n+1}$, the NLSE (1.1) will be solved in two splitting steps. One solves first

$$
i \partial_{t} \psi(\mathbf{x}, t)=-\frac{1}{2} \Delta \psi(\mathbf{x}, t), \quad \mathbf{x} \in \mathbb{R}^{d}, \quad t_{n} \leq t \leq t_{n+1},
$$

for the time step of length $\tau$, followed by solving

$$
i \partial_{t} \psi(\mathbf{x}, t)=[V(\mathbf{x})+\beta \varphi(\mathbf{x}, t)] \psi(\mathbf{x}, t), \quad \varphi(\mathbf{x}, t)=\left(U *|\psi|^{2}\right)(\mathbf{x}, t), \quad \mathbf{x} \in \mathbb{R}^{d}, \quad t_{n} \leq t \leq t_{n+1},
$$

for the same time step. For $t \in\left[t_{n}, t_{n+1}\right]$, Eq. (4.2) leaves $|\psi|$ invariant in $t[5,9]$, i.e., $|\psi(\mathbf{x}, t)|=\left|\psi\left(\mathbf{x}, t_{n}\right)\right|$, and thus $\varphi$ is time invariant, i.e., $\varphi(\mathbf{x}, t)=\varphi\left(\mathbf{x}, t_{n}\right):=\varphi^{n}(\mathbf{x})$, therefore it becomes

$$
i \partial_{t} \psi(\mathbf{x}, t)=\left[V(\mathbf{x})+\beta \varphi^{n}(\mathbf{x})\right] \psi(\mathbf{x}, t), \quad \varphi^{n}(\mathbf{x})=\left(U *\left|\psi^{n}\right|^{2}\right)(\mathbf{x}), \quad \mathbf{x} \in \mathbb{R}^{d}, \quad t_{n} \leq t \leq t_{n+1},
$$

where $\psi^{n}(\mathbf{x}):=\psi\left(\mathbf{x}, t_{n}\right)$, which immediately implies that

$$
\psi(\mathbf{x}, t)=e^{-i\left[V(\mathbf{x})+\beta \varphi^{n}(\mathbf{x})\right]\left(t-t_{n}\right)} \psi\left(\mathbf{x}, t_{n}\right), \quad \mathbf{x} \in \mathbb{R}^{d}, \quad t_{n} \leq t \leq t_{n+1} .
$$

Then an efficient and accurate numerical method can be designed by: (i) adopting a second-order Strang splitting [38] or a fourth-order time splitting method [40] to decouple the nonlinearity; (ii) truncating the problem on a bounded computational domain $\Omega$, and imposing the periodic BC on $\partial \Omega$ for the subproblem (4.1); (iii) discretizing (4.1) in space by the Fourier spectral method and integrating in time exactly; (iv) evaluating the nonlocal interaction $\varphi^{n}(\mathbf{x})$ in (4.4) by the algorithm via the NUFFT that discussed in previous sections, and integrating in time exactly for (4.4). The details are omitted here for brevity and this method is referred as the TS-NUFFT method.

For comparison, for the nonlocal interaction in $3 \mathrm{D} / 2 \mathrm{D}$, when the NUFFT in the above method is replaced by the standard FFT, we refer the method as TS-FFT. In addition, when the nonlocal interaction $\varphi^{n}(\mathbf{x})$ in (4.4) is reformulated as its equivalent PDE formulation (1.7)-(1.8) on $\Omega$ with homogeneous Dirichlet BC on $\partial \Omega$ and then discretized by the sine pseudospectral method with an evaluation of (4.1) via the sine spectral method and integrated in time exactly $[6,41]$, we refer it as TS-DST. 


\subsection{Numerical comparisons}

Again, in order to compare the TS-NUFFT method with the GF-DST method for computing the dynamics, we denote $\rho(\mathbf{x}, t)=|\psi(\mathbf{x}, t)|^{2}$ and $\varphi(\mathbf{x}, t)=\left(U *|\psi|^{2}\right)(\mathbf{x}, t)$ and introduce the errors

$$
\begin{aligned}
e_{\psi}^{h}(t) & :=\frac{\max _{\mathbf{x} \in \Omega^{h}}\left|\psi(\mathbf{x}, t)-\psi_{h}^{n}(\mathbf{x})\right|}{\max _{\mathbf{x} \in \Omega^{h}}|\psi(\mathbf{x}, t)|}, \quad e_{\varphi}^{h}(t):=\frac{\max _{\mathbf{x} \in \Omega^{h}}\left|\varphi(\mathbf{x}, t)-\varphi_{h}^{n}(\mathbf{x})\right|}{\max _{\mathbf{x} \in \Omega^{h}}|\varphi(\mathbf{x}, t)|}, \\
e_{\rho}^{h}(t) & :=\frac{\max _{\mathbf{x} \in \Omega^{h}}\left|\rho(\mathbf{x}, t)-\rho_{h}^{n}(\mathbf{x})\right|}{\max _{\mathbf{x} \in \Omega^{h}}|\rho(\mathbf{x}, t)|}, \quad t=t_{n}, \quad n \geq 0,
\end{aligned}
$$

where $\psi_{h}^{n}(\mathbf{x}), \varphi_{h}^{n}(\mathbf{x})$ and $\rho_{h}^{n}(\mathbf{x})$ are obtained numerically by a numerical method as the approximations of $\psi(\mathbf{x}, t), \varphi(\mathbf{x}, t)$ and $\rho(\mathbf{x}, t)$ at $t=t_{n}$, respectively with a given mesh size $h$ and a very small time step $\tau>0$. The external potential in (1.1) and the initial data in (1.3) are chosen as

$$
V(\mathbf{x})=\frac{|\mathbf{x}|^{2}}{2}, \quad \psi(\mathbf{x}, 0)=\psi_{0}(\mathbf{x})=e^{-\frac{|\mathbf{x}|^{2}}{2}}, \quad \mathbf{x} \in \mathbb{R}^{d} \text { with } d=3 \text { or } 2 .
$$

In the comparisons, the "exact" solution $\psi(\mathbf{x}, t)$ (and thus $\varphi(\mathbf{x}, t)$ and $\rho(\mathbf{x}, t)$ ) was obtained numerically via the TS-NUFFT method on a large enough domain $\Omega$ with very small enough mesh size $h$ and time step $\tau$. In our computations, we use the fourth-order time-splitting method for time integration [40].

Example 4.1: The NLSE with the $3 D$ Coulomb interaction. Here $d=3$ and $U(\mathbf{x})=U_{\mathrm{Cou}}(\mathbf{x})$ in (1.1)(1.2). The problem is solved numerically on a bounded computational domain $\Omega=[-8,8]^{3}$ with time step $\tau=10^{-3}$ and different mesh size $h$. Table 13 list the errors of the wave-function, the density and the 3D Coulomb interaction at $t=1 / 8$ obtained by the TS-NUFFT and TS-DST methods for different mesh size $h$ and interaction constant $\beta$.

Table 13: Errors of the wave-function and the nonlocal interaction at $t=1 / 8$ for the NLSE with the 3D Coulomb interaction.

\begin{tabular}{c|c|llll}
\hline \multicolumn{2}{c|}{ TS-NUFFT } & $h=1$ & $h=1 / 2$ & $h=1 / 4$ & $h=1 / 8$ \\
\hline$e_{\psi}^{h}(1 / 8)$ & $\beta=-5$ & $5.461 \mathrm{E}-03$ & $1.011 \mathrm{E}-05$ & $9.297 \mathrm{E}-12$ & $1.492 \mathrm{E}-13$ \\
& $\beta=5$ & $3.997 \mathrm{E}-03$ & $7.879 \mathrm{E}-06$ & $6.959 \mathrm{E}-12$ & $1.348 \mathrm{E}-13$ \\
\hline$e_{\varphi}^{h}(1 / 8)$ & $\beta=-5$ & $7.890 \mathrm{E}-03$ & $4.466 \mathrm{E}-06$ & $4.745 \mathrm{E}-12$ & $6.992 \mathrm{E}-14$ \\
& $\beta=5$ & $6.563 \mathrm{E}-03$ & $2.828 \mathrm{E}-06$ & $1.081 \mathrm{E}-12$ & $6.872 \mathrm{E}-14$ \\
\hline \hline \multicolumn{2}{c}{ TS-DST } & $h=1$ & $h=1 / 2$ & $h=1 / 4$ & $h=1 / 8$ \\
\hline$e_{\psi}^{h}(1 / 8)$ & $\beta=-5$ & $2.561 \mathrm{E}-02$ & $3.024 \mathrm{E}-02$ & $3.025 \mathrm{E}-02$ & $3.025 \mathrm{E}-02$ \\
& $\beta=5$ & $2.753 \mathrm{E}-02$ & $3.024 \mathrm{E}-02$ & $3.025 \mathrm{E}-02$ & $3.025 \mathrm{E}-02$ \\
\hline$e_{\rho}^{h}(1 / 8)$ & $\beta=-5$ & $5.567 \mathrm{E}-03$ & $1.444 \mathrm{E}-05$ & $2.397 \mathrm{E}-07$ & $2.441 \mathrm{E}-07$ \\
& $\beta=5$ & $5.590 \mathrm{E}-03$ & $1.416 \mathrm{E}-05$ & $2.560 \mathrm{E}-07$ & $2.568 \mathrm{E}-07$ \\
$e_{\varphi}^{h}(1 / 8)$ & $\beta=-5$ & $1.099 \mathrm{E}-01$ & $1.099 \mathrm{E}-01$ & $1.099 \mathrm{E}-01$ & $1.099 \mathrm{E}-01$ \\
& $\beta=5$ & $1.117 \mathrm{E}-01$ & $1.117 \mathrm{E}-01$ & $1.117 \mathrm{E}-01$ & $1.117 \mathrm{E}-01$ \\
\hline
\end{tabular}

Example 4.2: The NLSE with the $2 D$ Coulomb interaction. Here $d=2$ and $U(\mathbf{x})=U_{\mathrm{Cou}}(\mathbf{x})$ in (1.1)-(1.2). The problem is solved numerically on a bounded computational domain $\Omega=[-16,16]^{2}$ with time step $\tau=10^{-4}$ and different mesh size $h$. Table 14 shows the errors of the wave-function and the 2D Coulomb interaction at $t=0.5$ obtained by the TS-NUFFT and TS-DST methods for different mesh size $h$ and interaction constant $\beta$.

Example 4.3: The NLSE with the $2 D$ Poisson potential. Here $d=2$ and $U(\mathbf{x})=U_{\text {Lap }}(\mathbf{x})$ in (1.1)-(1.2). Again, the problem is solved numerically on a bounded computational domain $\Omega=[-16,16]^{2}$ with time step $\tau=10^{-4}$ and different mesh size $h$. Table 14 shows the errors of the wave-function and the 2D Coulomb interaction at $t=0.5$ obtained by the TS-NUFFT method for different mesh size $h$ and interaction constant 
Table 14: Errors of the wave-function and the nonlocal interaction at $t=0.5$ for the NLSE with the 2D Coulomb interaction.

\begin{tabular}{c|c|llll}
\hline \multicolumn{2}{c|}{ TS-NUFFT $(L=16)$} & $h=1$ & $h=1 / 2$ & $h=1 / 4$ & $h=1 / 8$ \\
\hline$e_{\psi}^{h}(0.5)$ & $\beta=-5$ & $1.582 \mathrm{E}-01$ & $7.468 \mathrm{E}-03$ & $4.746 \mathrm{E}-06$ & $2.954 \mathrm{E}-12$ \\
& $\beta=5$ & $5.118 \mathrm{E}-02$ & $7.756 \mathrm{E}-04$ & $2.476 \mathrm{E}-10$ & $1.268 \mathrm{E}-12$ \\
\hline$e_{\varphi}^{h}(0.5)$ & $\beta=-5$ & $2.219 \mathrm{E}-02$ & $4.242 \mathrm{E}-03$ & $4.169 \mathrm{E}-06$ & $3.756 \mathrm{E}-12$ \\
\hline \hline \multicolumn{2}{r|}{ TS-DST $(L=16)$} & $3.235 \mathrm{E}-02$ & $2.451 \mathrm{E}-04$ & $3.117 \mathrm{E}-11$ & $7.586 \mathrm{E}-13$ \\
\hline$e_{\psi}^{h}(0.5)$ & $\beta=-5$ & $h=1$ & $h=1 / 2$ & $h=1 / 4$ & $h=1 / 8$ \\
\hline & $\beta=5$ & $1.175 \mathrm{E}-01$ & $5.576 \mathrm{E}-02$ & $6.311 \mathrm{E}-02$ & $6.312 \mathrm{E}-02$ \\
$e_{\varphi}^{h}(0.5)$ & $\beta=-5$ & $6.477 \mathrm{E}-02$ & $6.308 \mathrm{E}-02$ & $6.313 \mathrm{E}-02$ & $6.313 \mathrm{E}-02$ \\
\hline \hline \multicolumn{2}{r|}{ TS-DST $(h=1 / 8)$} & $4.286 \mathrm{E}-02$ & $2.449 \mathrm{E}-02$ & $2.449 \mathrm{E}-02$ & $2.449 \mathrm{E}-02$ \\
\hline$e_{\psi}^{h}(0.5)$ & $\beta=-5$ & $6.854 \mathrm{E}-02$ & $4.412 \mathrm{E}-02$ & $4.455 \mathrm{E}-02$ & $4.478 \mathrm{E}-02$ \\
\hline$e_{\varphi}^{h}(0.5)$ & $\beta=5$ & $L=8$ & $L=16$ & $L=32$ & $L=64$ \\
\hline & $\beta=-5$ & $1.263 \mathrm{E}-01$ & $6.312 \mathrm{E}-02$ & $3.156 \mathrm{E}-02$ & $1.578 \mathrm{E}-02$ \\
& $\beta=5$ & $4.264 \mathrm{E}-01$ & $6.313 \mathrm{E}-02$ & $3.156 \mathrm{E}-02$ & $1.578 \mathrm{E}-02$ \\
\hline
\end{tabular}

Table 15: Errors of the wave-function and the Poisson potential at $t=0.5$ for the NLSE with the 2D Poisson potential.

\begin{tabular}{l|l|llll}
\hline \multicolumn{2}{c|}{ TS-NUFFT } & $h=1$ & $h=1 / 2$ & $h=1 / 4$ & $h=1 / 8$ \\
\hline$e_{\psi}^{h}(0.5)$ & $\beta=-5$ & $5.833 \mathrm{E}-02$ & $2.599 \mathrm{E}-04$ & $3.211 \mathrm{E}-09$ & $7.524 \mathrm{E}-13$ \\
& $\beta=5$ & $2.658 \mathrm{E}-02$ & $9.083 \mathrm{E}-05$ & $3.395 \mathrm{E}-12$ & $1.124 \mathrm{E}-12$ \\
\hline$e_{\varphi}^{h}(0.5)$ & $\beta=-5$ & $1.329 \mathrm{E}-02$ & $8.840 \mathrm{E}-05$ & $1.072 \mathrm{E}-09$ & $3.974 \mathrm{E}-13$ \\
& $\beta=5$ & $4.645 \mathrm{E}-03$ & $2.805 \mathrm{E}-06$ & $8.322 \mathrm{E}-13$ & $5.821 \mathrm{E}-13$ \\
\hline
\end{tabular}

$\beta$. We remark here that the TS-DST method is not applicable for this case [35, 41], therefore here we only present the results for the TS-NUFFT method.

From Tables 13-15 and additional numerical results not shown here for brevity, we can draw the following conclusions: (i) The TS-DST, if applicable, can not resolve the wave-function or the potential very accurately, while the TS-NUFFT achieves the spectral accuracy; (ii) The saturated accuracy by TS-DST decreases as the computation domain increases; (iii) As long as for the physical observables, e.g., the density $\rho$, are concerned, the TS-DST method can still capture reasonable accuracy (cf. Tab. 13).

\subsection{Applications}

To further demonstrate the efficiency and accuracy of the numerical method via the NUFFT, we simulate the long-time dynamics of the 2D NLSE with the Coulomb interaction, i.e., $d=2$ and $U(\mathbf{x})=U_{\text {Cou }}(\mathbf{x})$ and $\beta=5$ in (1.1)-(1.2), and a honeycomb external potential [9, 20] defined as

$$
V(\mathbf{x})=10\left[\cos \left(\mathbf{b}_{\mathbf{1}} \cdot \mathbf{x}\right)+\cos \left(\mathbf{b}_{\mathbf{2}} \cdot \mathbf{x}\right)+\cos \left(\left(\mathbf{b}_{\mathbf{1}}+\mathbf{b}_{\mathbf{2}}\right) \cdot \mathbf{x}\right)\right], \quad \mathbf{x}=(x, y)^{T} \in \mathbb{R}^{2},
$$

with $\mathbf{b}_{\mathbf{1}}=\frac{\pi}{4}(\sqrt{3}, 1)^{T}$ and $\mathbf{b}_{\mathbf{2}}=\frac{\pi}{4}(-\sqrt{3}, 1)^{T}$. This example can be formally used to describe the dynamics of the electrons in a graphene. The initial data in (1.3) is taken as $\psi_{0}(x, y)=e^{-\left(x^{2}+y^{2}\right) / 2}$ for $\mathbf{x} \in \mathbb{R}^{2}$ and the problem is solved numerically on $\Omega=[-32,32]^{2}$ by using the TS-NUFFT with mesh size $h=\frac{1}{16}$ and time step $\tau=10^{-4}$. Figure 3 shows the contour plots of the density $\rho(x, y, t)$ at different times. 

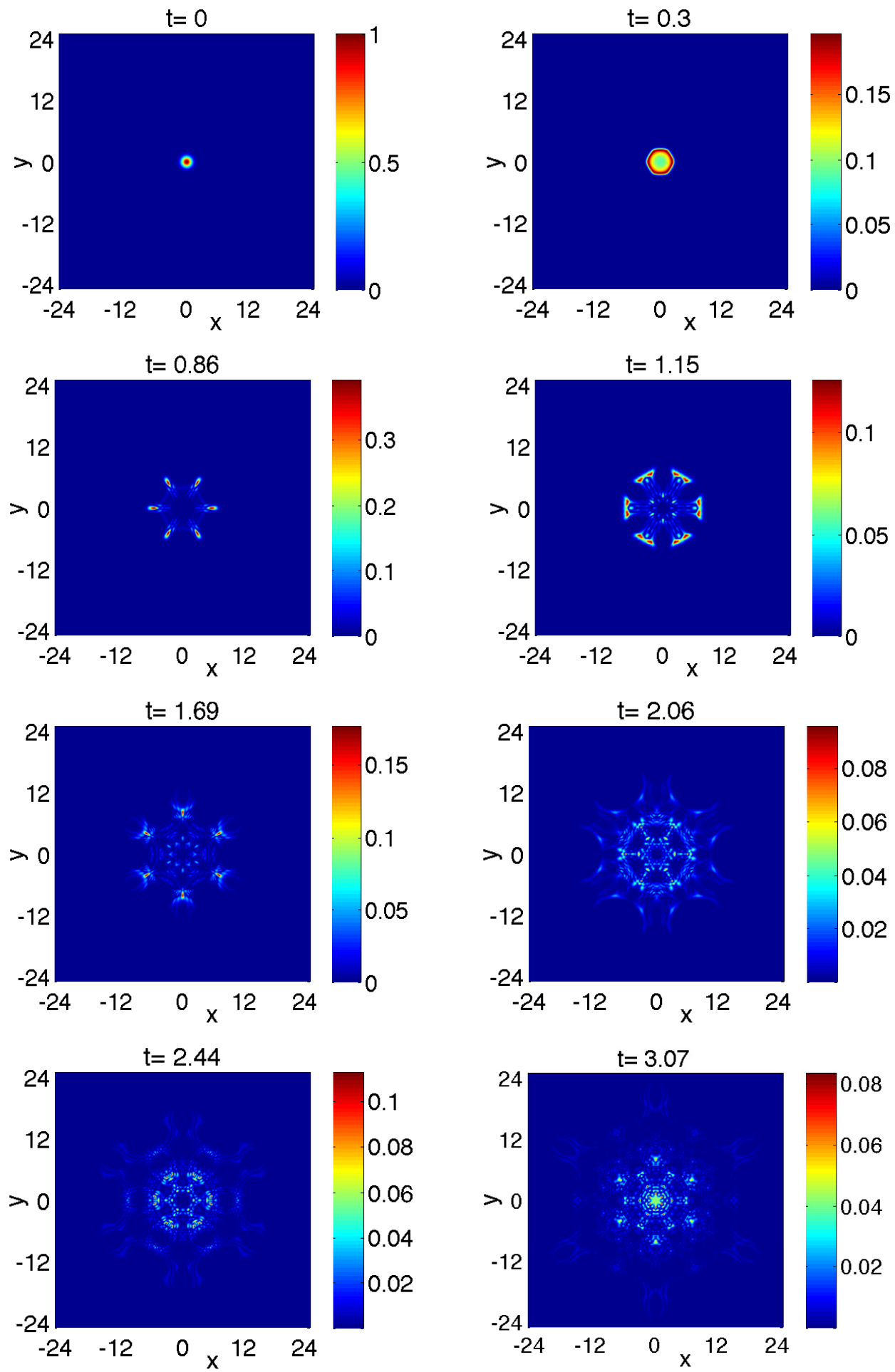

Figure 3: Contour plots of the density $\rho(x, y, t)$ of the NLSE with the Coulomb interaction and a honeycomb potential in $2 \mathrm{D}$ at different times. 


\section{Conclusion}

An efficient and accurate numerical method via the NUFFT was proposed for the fast evaluation of different nonlocal interactions including the Coulomb interactions in $3 \mathrm{D} / 2 \mathrm{D}$ and the interaction kernel taken as either the Green's function of the Laplace operator in 3D/2D/1D or nonlocal interaction kernels in 2D/1D obtained from the 3D Schrödinger-Poisson system under strongly external confining potentials via dimension reduction. The method was compared extensively with those existing numerical methods and was demonstrated that it can achieve much more accurate numerical results, especially on a smaller computational domain and/or with anisotropic interaction density. Also, efficient and accurate numerical methods were presented for computing the ground state and dynamics of the NLSE with nonlocal interactions. These methods combine the NUFFT based method for interaction evaluation with the normalized gradient flow method for ground state computation and/or time-splitting Fourier pseudospectral method for dynamics simulation, respectively. Extensive numerical comparisons with other existing methods were then carried out. Numerical results showed clearly that the NUFFT based methods outperform those existing methods in terms of accuracy and efficiency, especially when the computational domain is chosen smaller and/or the solution is anisotropic.

\section{Acknowledgments}

Part of this work was done when the authors were visiting Beijing Computational Science Research Center in the summer of 2014. We acknowledge support from the Ministry of Education of Singapore grant R-146-000-196-112 (W. Bao), the National Science Foundation under grant DMS-1418918 (S. Jiang), the French ANR-12-MONU-0007-02 BECASIM (Q. Tang) and the Austrian Science Foundation (FWF) under grant No. F41 (project VICOM), grant No. I830 (project LODIQUAS), grant no. FR 15/2014 ("WTZ" France by OEAD) and the Austrian Ministry of Science and Research via its grant for the WPI (Y. Zhang). The computation results presented have been achieved by using the Vienna Scientific Cluster.

\section{References}

[1] M. Abbamowitz and I. A. Stegun, Handbook of Mathematical Functions, Dover, 1965.

[2] X. Antoine, W. Bao and C. Besse, Computational methods for the dynamics of the nonlinear Schrödinger/Gross-Pitaevskii equations, Comput. Phys. Commun. 184 (2013) 2621-2633.

[3] X. Antoine, R. Duboscq, Robust and efficient preconditioned Krylov spectral solvers for computing the ground states of fast rotating and strongly interacting Bose-Einstein condensates, J. Comput. Phys. 258 (2014) 509-523.

[4] W. Bao, N. Ben Abdallah and Y. Cai, Gross-Pitaevskii-Poisson equations for dipolar Bose-Einstein condensate with anisotropic confinement, SIAM J. Math. Anal. 44 (2012) 1713-1741.

[5] W. Bao and Y. Cai, Mathematical theory and numerical methods for Bose-Einstein condensation, Kinet. Relat. Mod. 6 (2013) 1-135.

[6] W. Bao, Y. Cai and H. Wang, Efficient numerical methods for computing ground states and dynamics of dipolar BoseEinstein condensates, J. Comput. Phys. 229 (2010) 7874-7892.

[7] W. Bao, I-L. Chern and F. Lim, Efficient and spectrally accurate numerical methods for computing ground and first excited states in Bose-Einstein condensates, J. Comput. Phys. 219 (2006) 836-854.

[8] W. Bao and Q. Du, Computing the ground state solution of Bose-Einstein condensates by a normalized gradient flow, SIAM J. Sci. Comput. 25 (2004) 1674-1697.

[9] W. Bao, H. Jian, N. J. Mauser and Y. Zhang, Dimension reduction of the Schrödinger equation with Coulomb and anisotropic confining potentials, SIAM J. Appl. Math. 73 (6) (2013) 2100-2123.

[10] W. Bao, D. Marahrens, Q. Tang and Y. Zhang, A simple and efficient numerical method for computing the dynamics of rotating Bose-Einstein condensates via a rotating Lagrangian coordinate, SIAM J. Sci. Comput. 35 (6) (2013) A2671A2695.

[11] W. Bao, N. J. Mauser and H. P. Stimming, Effective one particle quantum dynamics of electrons: A numerical study of the Schrödinger-Poisson-X $\alpha$ model, Comm. Math. Sci. 1 (2003) 809-831.

[12] C. Bardos, L. Erdős, F. Golse, N. J. Mauser and H.-T. Yau, Derivation of the Schrödinger-Poisson equation from the quantum $N$-particle Coulomb problem, C. R. Math. Acad. Sci. Paris 334 (6) (2002) 515-520.

[13] C. Bardos, F. Golse and N. J. Mauser, Weak coupling limit of the $N$-particle Schrödinger equation, Methods Appl. Anal. 7(2) (2000) 275-293.

[14] N. Ben Abdallah, F. Méhats and O. Pinaud, Adiabatic approximation of the Schrödinger-Poisson system with a partial confinement, SIAM J. Math. Anal. 36 (2005) 986-1013. 
[15] O. Bokanowski, J. L. López and J. Soler, On a exchange interaction model for quantum transport: The SchrödingerPoisson-Slater system, Math. Model Methods Appl. Sci. 12 (10) (2003) 1397-1412.

[16] O.P. Bruno and L.A. Kunyansky, A fast, high-order algorithm for the solution of surface scattering problems: basic implementation, tests, and applications, J. Comput. Phys. 169 (2001) 80-110.

[17] Y. Cai, M. Rosenkranz, Z. Lei and W. Bao, Mean-field regime of trapped dipolar Bose-Einstein condensates in one and two dimensions, Phys. Rev. A 82 (2010) 043623.

[18] I. Catto, J. Dolbeault, O. Sánchez and J. Soler, Existence of steady states for the Maxwell-Schrödinger-Poisson system: exploring the applicability of the concentration-compactness principle, Math. Model Methods Appl. Sci. 23 (10) (2013) 1915-1938.

[19] T. Cazenave, Semilinear Schrödinger Equations, Courant Lecture Notes in Mathematics, vol. 10, New York University Courant Institute of Mathematical Sciences AMS, 2003.

[20] Z. Chen and B. Wu, Bose-Einstein condensate in a honeycomb optical lattice: fingerprint of superfluidity at the Dirac point, Phys. Rev. Lett. 107 (2011) 065301.

[21] X. Dong, A short note on simplified pseudospectral methods for computing ground state and dynamics of spherically symmetric Schrödinger-Poisson-Slater system, J. Comput. Phys. 230 (2011) 7917-7922.

[22] A. Dutt and V. Rokhlin, Fast Fourier transforms for nonequispaced data, SIAM J. Sci. Comput. 14 (1993) $1368-1393$.

[23] L. Erdös and H.-T. Yau, Derivation of the nonlinear Schrödinger equation from a many body Coulomb system, Adv. Theor. Math. Phys. 5 (2001) 1169-1205.

[24] F. Ethridge and L. Greengard, A new fast-multipole accelerated Poisson solver in two dimensions, SIAM J. Sci. Comput. 23 (3) (2001) 741-760.

[25] Z. Gimbutas, L. Greengard and M. Minion, Coulomb interactions on planar structures: inverting the square root of the Laplacian, SIAM J. Sci. Comput. 22 (6) (2000) 2093-2108.

[26] L. Greengard and J.Y. Lee, Accelerating the nonuniform fast Fourier transform, SIAM Rev. 46 (2004) $443-454$.

[27] L. Greengard and V. Rokhlin, A new version of the fast multipole method for the Laplace equation in three dimensions, Acta Numerica 6 (1997) 229-269.

[28] H. Han and W. Bao, Error estimates for the finite element approximation of problems in unbounded domains, SIAM J. Numer. Anal. 37 (2000) 1101-1119.

[29] S. Jiang, L. Greengard and W. Bao, Fast and accurate evaluation of nonlocal Coulomb and dipole-dipole interactions via the nonuniform FFT, SIAM J. Sci. Comput. 36 (2014) B777-B794.

[30] S. Jin, H. Wu and X. Yang, A numerical study of the Gaussian beam methods for one-dimensional Schrödinger-Poisson equations, J. Comput. Math 28 (2010) 261-272.

[31] C. Lubich, On splitting methods for Schrödinger-Poisson and cubic nonlinear Schrödinger equations, Math. Comp. 77 (2008) 2141-2153.

[32] P. A. Markowich, C. Ringhofer and C. Schmeiser, Semiconductor Equations, Springer-Verlag, 1990.

[33] S. Masaki, Energy solution to a Schrödinger-Poisson system in the two-dimensional whole space, SIAM J. Math. Anal. 43 (2011) 2719-2731.

[34] F. Méhats, Analysis of a quantum subband model for the transport of partially confined charged particles, Monatch. Math. 147 (2006) 43-73.

[35] N. J. Mauser and Y. Zhang, Exact artificial boundary condition for the Poisson equation in the simulation of the 2D Schrödinger-Poisson system, Commun. Comput. Phys. 16 (3) (2014) 764-780.

[36] Ó. Sánchez and J. Soler, Long time dynamics of the Schrödinger-Poisson-Slater systems, J. Statist. Phys. 114 (2004) 179-204.

[37] H. P. Stimming and Y. Zhang, A novel nonlocal potential solver based on nonuniform FFT for efficient simulation of the Davey-Stewartson equations, arXiv:1409.2014.

[38] G. Strang, On the construction and comparision of difference schemes, SIAM J. Numer. Anal. 5 (1968) 505-517.

[39] M. Thalhammer, High-order exponential operator splitting methods for time-dependent Schrödinger equations, SIAM J. Numer. Anal. 46 (2008) 2022-2038.

[40] H. Yoshida, Construction of higher order symplectic integrators, Phys. Lett. A. 150 (1990) $262-268$.

[41] Y. Zhang and X. Dong, On the computation of ground states and dynamics of Schrödinger-Poisson-Slater system, J. Comput. Phys. 230 (2011) 2660-2676. 